\title{
Finite-Rank Multivariate-Basis Expansions of the Resolvent Operator as a Means of Solving the Multivariable Lippmann-Schwinger Equation for Two-Particle Scattering
}

Received: 24 January 2014 / Accepted: 3 April 2014

(C) Springer-Verlag Wien 2014

\begin{abstract}
Finite-rank expansions of the two-body resolvent operator are explored as a tool for calculating the full three-dimensional two-body $T$-matrix without invoking the partial-wave decomposition. The separable expansions of the full resolvent that follow from finite-rank approximations of the free resolvent are employed in the Low equation to calculate the T-matrix elements. The finite-rank expansions of the free resolvent are generated via projections onto certain finite-dimensional approximation subspaces. Types of operator approximations considered include one-sided projections (right or left projections), tensor-product (or outer) projection and inner projection. Boolean combination of projections is explored as a means of going beyond tensor-product projection. Two types of multivariate basis functions are employed to construct the finitedimensional approximation spaces and their projectors: (i) Tensor-product bases built from univariate local piecewise polynomials, and (ii) multivariate radial functions. Various combinations of approximation schemes and expansion bases are applied to the nucleon-nucleon scattering employing a model two-nucleon potential. The inner-projection approximation to the free resolvent is found to exhibit the best convergence with respect to the basis size. Our calculations indicate that radial function bases are very promising in the context of multivariable integral equations.
\end{abstract}

\section{Introduction}

The usual line of approach to quantum scattering calculations has almost always been through the elimination of angular variables via expansions over angular-momentum states. Certain drawbacks of this strategy have been noted in recent years, especially for high-energy collisions and within the context of few-body problems. As a result, computational methods that avoid the traditional decomposition of wave functions and scattering amplitudes into partial waves have been explored recently by a number of groups [1-12]. Various direct multivariable methods have been investigated for the solution of two-body Lippmann Schwinger (LS) equation. Most studies [1,3,5-9] employed the Nystrom method (i.e., discretization of the integral equation via a suitable multi-variate quadrature) [13]. Although the Nystrom method can produce very accurate results, the matrix dimensions in the multi-variable Nystrom approach can grow very fast to computationally prohibitive levels. Multi-variable methods that lead to a reduction in the matrix sizes are therefore of considerable interest.

A variety of weighted-residual methods, such as Galerkin [2,4], collocation [11], Schwinger variational methods [11], with various choices of multivariate bases have been considered. Among these, multivariable version of the Schwinger variational method (with local interpolation polynomials as the expansion basis) and some of its variants have been shown to be quite effective [11]. In a similar vein, a multivariate Bateman interpolation of the momentum-space kernel of the potential have proved to be a relatively simple and effective 
method [12]. It is interesting to note that both these methods can also be viewed as the result of certain finite-rank separable approximations of the interaction potential $[11,14]$.

An alternative to the direct solution of the LS equation for $T$-matrix is to solve the analog of the LS equation for the resolvent and then to use the Low equation to construct the T-matrix. As separable expansions of the interaction potential lead to separable expansions of the T-operator, separable expansions of the free resolvent give rise to separable expansions of the full resolvent. Hence analogs of projection schemes that are used to obtain separable expansions of the interaction potential can also be employed for the free resolvent. Given a finite-dimensional approximation space and its (orthogonal) projector $\mathcal{P}$, we can introduce for an operator $F$ the following approximations on this subspace:

$$
\begin{array}{rlrl}
F^{O} & =\mathcal{P} F \mathcal{P} & & \text { tensor-product (or outer) projection } \\
F^{L} & =\mathcal{P} F & & \text { left projection } \\
F^{R} & =F \mathcal{P} & & \text { right projection } \\
F^{I} & =F \mathcal{P}(\mathcal{P} F \mathcal{P})^{-1} \mathcal{P} F & \text { inner projection }
\end{array}
$$

where the terminology of outer and inner projections for $F^{O}$ and $F^{I}$, respectively, is adopted from Ref. [15].

An important tool in multivariate interpolation and approximation by projections is the Boolean combination of projection operators [16-18]. This idea gives rise to the so-called blending-function methods [16-19] for interpolation of multivariate functions. An early application of this idea to scattering equations can be found in [20] where the bivariate kernel of an integral equation is expanded in terms of blending functions (which are sections of the bivariate kernel). In our present context, the Boolean combination of right, left and outer projections is defined as

$$
F^{B}=\mathcal{P} F+F \mathcal{P}-\mathcal{P} F \mathcal{P}=F^{L}+F^{R}-F^{O} .
$$

The use of these five type of projections for the free resolvent $G_{0}$ in suitably constructed approximation spaces lead to finite-rank expansions for the full resolvent $G$, which can then be used to calculate the T-matrix. We find that, for a given basis (and the $\mathcal{P}$ associated with it), inner and Boolean projections provide the most promising computational schemes. We note in passing that some of these projection schemes are intimately related to Schwinger-type variational methods for the resolvent [14,21-26]. All previous applications of these methods, however, have been on single-variable scattering equations that result from expansions over internal states and partial wave analysis.

Standard approach to building a multivariate approximation space is through tensor-product of univariatebases. We use this approach with local piecewise polynomials defined over a grid in each univariate variable as employed, e.g., in finite-element methods [27,28]. However, tensor-product schemes suffer from the curse of dimensionality. Radial basis functions (rbf's) [16,17,29-33] have emerged in recent years as powerful tools in multivariate interpolation and approximation. Interpolation of scattered data $[29,30]$ and meshless methods for partial differential equations [31-33] are two areas where rbf's have become a standard tool. We have employed a variety of rbf's to build the projection operators needed for the approximation of two- and three-dimensional resolvents. Our results are very promising.

Plan of this article is as follows: In Sect. 2, we fix notation, introduce finite-rank approximations for the free resolvent $G_{0}$, and derive the working equations for the computation of $T$-matrix elements. In Sect. 3, a new projection approximation based on Boolean combination of left, right and outer projections is formulated. Section 4 discusses the two-variable versions of the equations for central potentials that follow from the elimination of the azimuthal angle. In Sect. 5, tensor-product and radial bases for multivariate approximation are introduced. Results of two- and three-dimensional calculations for a model two-nucleon potential are presented and compared in Sect. 6 for different methods and bases. Our concluding remarks are made in Sect. 7.

\section{Finite-Rank Resolvent Approximations}

The basic equation for two-particle scattering is the Lippmann-Schwinger (LS) equation which reads in operator form

$$
T(z)=V+V G_{0}(z) T(z)
$$


where $T$ is the transition operator, $V$ the two-body potential, $G_{0}=\left(z-H_{0}\right)^{-1}$ the free resolvent, with $H_{0}$ being the free hamiltonian and $z$ the (complex) energy of the two-body system. Working in the center-of-mass frame, the eigenstates of $H_{0}$ will be denoted as $\mid \mathbf{q}>$, viz., $H_{0}\left|\mathbf{q}>=\left(q^{2} / 2 \mu\right)\right| \mathbf{q}>$. For on-shell scattering, $z=E+i 0$, with $E=q_{0}^{2} / 2 \mu$, where $\mu$ is the reduced mass. The quantities of computational interest are the momentum-space matrix elements $T\left(\mathbf{q}, \mathbf{q}^{\prime} ; z\right)\left(\equiv\left\langle\mathbf{q}|T(z)| \mathbf{q}^{\prime}\right\rangle\right)$ which satisfy the three-dimensional integral equation

$$
T\left(\mathbf{q}, \mathbf{q}^{\prime}\right)=V\left(\mathbf{q}, \mathbf{q}^{\prime}\right)+\int \mathrm{d} \mathbf{q}^{\prime \prime} \frac{V\left(\mathbf{q}, \mathbf{q}^{\prime \prime}\right) T\left(\mathbf{q}^{\prime \prime}, \mathbf{q}^{\prime}\right)}{z-q^{\prime \prime 2} / 2 \mu}
$$

where energy dependence of $T$-matrix elements has been suppressed.

The formal solution of Eq. (1) is the Low equation

$$
T(z)=V+V G(z) V
$$

where $G=(z-H)^{-1}$ is the full resolvent, with $H=H_{0}+V$ being the full Hamiltonian. The full resolvent satisfies

$$
G(z)=G_{0}(z)+G_{0}(z) V G(z) .
$$

Equations (6) and (8) will be referred to as the T-LS and G-LS equations, respectively. As the T-LS equation reduces to algebraic equations for finite-rank potentials, the G-LS equation is similarly converted into a system of algebraic equations when $G_{0}$ is approximated by a finite-rank expansion.

The solutions to LS equations are typically sought within a finite-dimensional approximation space $\mathcal{S}_{\mathcal{A}}$. If we let $\mathcal{P}$ denote the orthogonal projector to this approximation space, the following finite-rank approximations for $G_{0}$ can be introduced

$$
\begin{aligned}
G_{0}^{O} & =\mathcal{P} G_{0} \mathcal{P}, \\
G_{0}^{L} & =\mathcal{P} G_{0} \\
G_{0}^{R} & =G_{0} \mathcal{P} \\
G_{0}^{I} & =G_{0} \mathcal{P}\left(\mathcal{P} G_{0} \mathcal{P}\right)^{-1} \mathcal{P} G_{0} .
\end{aligned}
$$

Following the terminology of Lowdin [15], $G_{0}^{O}$ will be referred to as the outer-projection (OP) approximation, while $G_{0}^{I}$ as the inner-projection (IP) approximation of $G_{0}$. The one-sided projections $G_{0}^{L}$ and $G_{0}^{R}$ are termed as left-projection (LP) and right-projection (RP) approximations, respectively. The exact solutions of the G-LS equation with these separable approximations of $G_{0}$ read

$$
\begin{aligned}
G^{O} & =\mathcal{P} G_{0} \mathcal{P}\left[\mathcal{P}\left(G_{0}-G_{0} \mathcal{P} V \mathcal{P} G_{0}\right) \mathcal{P}\right]^{-1} \mathcal{P} G_{0} \mathcal{P}, \\
G^{L} & =\mathcal{P}\left[\mathcal{P}\left(I-G_{0} V\right) \mathcal{P}\right]^{-1} \mathcal{P} G_{0} \\
G^{R} & =G_{0} \mathcal{P}\left[\mathcal{P}\left(1-V G_{0}\right) \mathcal{P}\right]^{-1} \mathcal{P} \\
G^{I} & =G_{0} \mathcal{P}\left[\mathcal{P}\left(G_{0}-G_{0} V G_{0}\right) \mathcal{P}\right]^{-1} \mathcal{P} G_{0}
\end{aligned}
$$

Use of these approximations in the Low equation leads to the approximate $T$-matrices:

$$
T^{A}=V+V G^{A} V,
$$

where $A=O, L, R$, or $I$. We note that the same $T$-matrix approximations $T^{A}$ embodied in Eq. 18 would follow from using $G_{0}^{A}$, with $A=O, L, R$ or $I$, directly in the T-LS equation. We also note that the IP-approximation $G^{I}$ is equivalent to the solution of the G-LS equation via a Schwinger-type variational principle [21-23] (which in this context is sometimes referred to as the Newton variational principle [24,26]), while LP-approximation $G^{L}$ corresponds to solution of the G-LS equation via the Galerkin method [11].

As the resolvent operators by themselves are not compact for $z=E+i 0$, a word of caution must be mentioned about the finite-rank expansions 10-13 and 14-17. In this article, these finite-rank expansions are meant to be used in contexts like $V G_{0} V$ and $V G V$. As shown by Lovelace in [34], the kernel $V G_{0}$ of the LS equation is compact for $z=E+i 0$ in a suitable Banach space (namely, the Banach space $C_{1}$ of bounded continuous differentiable functions with bounded continuous derivatives) for a fairly large class of potentials. Also, as shown in Refs. [35,36], for potentials that satify a certain mild condition (which, however, excludes the Coulomb potential), the symmetrized kernel $V^{1 / 2} G_{0}(z) V^{1 / 2}$, and hence $V G_{0}$, is compact in the limit $z=E+i 0$ in the Hilbert Space as well. Of course, it is a well-known fact that compact kernels can be 
uniformly approximated by finite-rank expansions. If we multiply the resolvent expansions 10-17 with $V$ from both sides, we can view the resulting expressions as finite-rank expansions of the compact operators $V G_{0} V$ or $V G V$.

We specify the approximation subspace $\mathcal{S}_{\mathcal{A}}$ by choosing a set $\left\{\varphi_{n}(\mathbf{q}), n=1,2, \ldots, N\right\}$ of basis functions. These multivariate basis functions are linearly independent, but not necessarily orthonormal. The projection operator onto the approximation subspace $\mathcal{S}_{\mathcal{A}}$ is given as

$$
\begin{aligned}
\mathcal{P} & =\Sigma_{n=1}^{N} \Sigma_{n^{\prime}=1}^{N}\left|\varphi_{n}>\left(\boldsymbol{\Delta}^{-1}\right)_{n, n^{\prime}}<\varphi_{n^{\prime}}\right| \\
& =\Sigma_{n=1}^{N}\left|\varphi_{n}><\bar{\varphi}_{n^{\prime}}\right|=\Sigma_{n=1}^{N}\left|\bar{\varphi}_{n}><\varphi_{n^{\prime}}\right|
\end{aligned}
$$

where $\boldsymbol{\Delta}$ is the overlap matrix, viz., $\boldsymbol{\Delta}_{n, n^{\prime}}=\left\langle\varphi_{n} \mid \varphi_{n^{\prime}}\right\rangle$ and $\bar{\varphi}_{n}(\mathbf{q})$ is the biorthogonal partner of $\varphi_{n}(\mathbf{q})$, viz., $\left|\bar{\varphi}_{n}>=\Sigma_{n^{\prime}=1}^{N}\right| \varphi_{n}^{\prime}>\left(\boldsymbol{\Delta}^{-1}\right)_{n^{\prime}, n}$.

Upon using the explicit form of the projector in Eq. (19), the $T$-matrix elements are obtained as

$$
\begin{aligned}
& T^{O}\left(\mathbf{q}, \mathbf{q}^{\prime}\right)=V\left(\mathbf{q}, \mathbf{q}^{\prime}\right)+\Sigma_{n} \Sigma_{n^{\prime}}\left\langle\mathbf{q}|V| \varphi_{n}\right\rangle \mathbf{D}_{n, n^{\prime}}^{O}\left\langle\varphi_{n^{\prime}}|V| \mathbf{q}^{\prime}\right\rangle, \\
& T^{L}\left(\mathbf{q}, \mathbf{q}^{\prime}\right)=V\left(\mathbf{q}, \mathbf{q}^{\prime}\right)+\Sigma_{n} \Sigma_{n^{\prime}}\left\langle\mathbf{q}|V| \varphi_{n}\right\rangle \mathbf{D}_{n, n^{\prime}}^{L}\left\langle\varphi_{n^{\prime}}\left|G_{0} V\right| \mathbf{q}^{\prime}\right\rangle, \\
& T^{R}\left(\mathbf{q}, \mathbf{q}^{\prime}\right)=V\left(\mathbf{q}, \mathbf{q}^{\prime}\right)+\Sigma_{n} \Sigma_{n^{\prime}}\left\langle\mathbf{q}\left|V G_{0}\right| \varphi_{n}\right\rangle \mathbf{D}_{n, n^{\prime}}^{R}\left\langle\varphi_{n^{\prime}}|V| \mathbf{q}^{\prime}\right\rangle, \\
& T^{I}\left(\mathbf{q}, \mathbf{q}^{\prime}\right)=V\left(\mathbf{q}, \mathbf{q}^{\prime}\right)+\Sigma_{n} \Sigma_{n^{\prime}}\left\langle\mathbf{q}\left|V G_{0}\right| \varphi_{n}\right\rangle \mathbf{D}_{n, n^{\prime}}^{I}\left\langle\varphi_{n^{\prime}}\left|G_{0} V\right| \mathbf{q}^{\prime}\right\rangle,
\end{aligned}
$$

where

$$
\begin{aligned}
& {\left[\left(\mathbf{D}^{O}\right)^{-1}\right]_{n, n^{\prime}}=\left(\mathbf{G}_{\mathbf{0}}^{-1}-\mathbf{\Delta}^{-1} \mathbf{V} \mathbf{\Delta}^{-1}\right)_{n n^{\prime}},} \\
& {\left[\left(\mathbf{D}^{L}\right)^{-1}\right]_{n, n^{\prime}}=\left\langle\varphi_{n}\left|I-G_{0} V\right| \varphi_{n^{\prime}}\right\rangle} \\
& {\left[\left(\mathbf{D}^{R}\right)^{-1}\right]_{n, n^{\prime}}=\left\langle\varphi_{n}\left|I-V G_{0}\right| \varphi_{n^{\prime}}\right\rangle} \\
& {\left[\left(\mathbf{D}^{I}\right)^{-1}\right]_{n, n^{\prime}}=\left\langle\varphi_{n}\left|G_{0}-G_{0} V G_{0}\right| \varphi_{n^{\prime}}\right\rangle}
\end{aligned}
$$

In Eq. (25), the matrices $\mathbf{G}_{\mathbf{0}}$ and $\mathbf{V}$ consist of the elements $\left(\mathbf{G}_{\mathbf{0}}\right)_{n n^{\prime}}=\left\langle\varphi_{n}\left|G_{0}\right| \varphi_{n^{\prime}}\right\rangle$ and $(\mathbf{V})_{n n^{\prime}}=\left\langle\varphi_{n}|V| \varphi_{n^{\prime}}\right\rangle$.

Correct handling of the singular integrals $\left\langle\varphi_{n}\left|G_{0}\right| \varphi_{n^{\prime}}\right\rangle,\left\langle\varphi_{n}\left|V G_{0}\right| \varphi_{n^{\prime}}\right\rangle,\left\langle\varphi_{n}\left|G_{0} V\right| \varphi_{n^{\prime}}\right\rangle$, and $\left\langle\varphi_{n}\left|V G_{0} V\right| \varphi_{n^{\prime}}\right\rangle$ is crucial for the computational implementation of these resolvent approximations. A subtraction procedure has been described in Ref. [11] for numerical treatment of such integrals.

\section{Boolean Combination of Projection Approximations}

In multivariate interpolation theory [16,17], the blending-function methods are used to go beyond the tensorproduct interpolation. Blending-type approach to the interpolation of a multivariate function employ as basis functions certain sections (or cuts) of the multivariate function and involve Boolean combination of interpolatory projections [16-19]. The same technique can be used in the context of multivariate approximation by orthogonal projectors.

To formulate the Boolean approximation, e.g., for 6-variate functions (in variables $\mathbf{q}$ and $\mathbf{q}^{\prime}$ ), we first distinguish between the orthogonal projector $\mathcal{P}$ onto approximation space $\mathcal{S}_{\mathcal{A}}$ for the vector-variable $\mathbf{q}$, and the orthogonal projector $\mathcal{P}^{\prime}$ onto the approximation space $\mathcal{S}_{\mathcal{A}}^{\prime}$ for the vector variable $\mathbf{q}^{\prime}$. These approximation spaces could in fact be chosen to be different, but in the present work $\mathcal{S}_{\mathcal{A}}^{\prime}$ is taken as a replica of $\mathcal{S}_{\mathcal{A}}$. The extensions of $\mathcal{P}$ and $\mathcal{P}^{\prime}$ to the space of two-vector-variable (6-variate) functions (like the potential kernel $\left.V\left(\mathbf{q}, \mathbf{q}^{\prime}\right)\right)$ are given as $\overline{\mathcal{P}} \equiv \mathcal{P} \otimes \mathcal{I}^{\prime}$ and $\overline{\mathcal{P}}^{\prime} \equiv I \otimes \mathcal{P}^{\prime}$, where $I$ and $I^{\prime}$ are the identity operators in the respective function spaces for the variables $\mathbf{q}$ and $\mathbf{q}^{\prime}$. The Boolean sum $\overline{\mathcal{P}} \oplus \overline{\mathcal{P}}^{\prime}$ is defined as $\overline{\mathcal{P}} \oplus \overline{\mathcal{P}}=\overline{\mathcal{P}}+\overline{\mathcal{P}}^{\prime}-\overline{\mathcal{P}} \overline{\mathcal{P}}^{\prime}$. As $\overline{\mathcal{P}}$ and $\overline{\mathcal{P}}^{\prime}$ commute, it is easily verified that $\overline{\mathcal{P}} \oplus \overline{\mathcal{P}}^{\prime}$ is a projector. If the multivariate function $F\left(\mathbf{q}, \mathbf{q}^{\prime}\right)$ is the 
momentum space kernel of an operator $F$, the Boolean approximation for this kernel function can be written as

$$
\begin{aligned}
\left(\overline{\mathcal{P}} \oplus \overline{\mathcal{P}}^{\prime}\right) F\left(\mathbf{q}, \mathbf{q}^{\prime}\right)= & \sum_{n} \varphi_{n}(\mathbf{q})\left\langle\bar{\varphi}_{n}|F| \mathbf{q}^{\prime}\right\rangle+\sum_{n^{\prime}}\left\langle\mathbf{q}|F| \bar{\varphi}_{n^{\prime}}\right\rangle \varphi_{n^{\prime}}^{*}\left(\mathbf{q}^{\prime}\right) \\
& -\sum_{n} \sum_{n^{\prime}} \varphi_{n}(\mathbf{q})\left\langle\bar{\varphi}_{n}|F| \bar{\varphi}_{n^{\prime}}\right\rangle \varphi_{n^{\prime}}^{*}\left(\mathbf{q}^{\prime}\right) .
\end{aligned}
$$

In operator form, $F^{B}=\mathcal{P} F+F \mathcal{P}-\mathcal{P} F \mathcal{P}$, where superscript $B$ stands for Boolean. Thus, the Boolean approximation is a particular combination of the left-, right- and outer-projections considered in the previous section. Other combinations like $(\mathcal{P} F+F \mathcal{P}) / 2$, or $(\mathcal{P} F+F \mathcal{P}+\mathcal{P} F \mathcal{P}) / 3$ are conceivable, but will not be pursued in the present paper.

To get further insight into the Boolean projection, we introduce an extended basis $\left\{\Phi_{1}, \Phi_{2}, \ldots, \Phi_{2 N}\right\}$, where $\Phi_{i}=\varphi_{i}$ and $\Phi_{N+i}=F \mid \bar{\varphi}_{i}>$ for $i=1, \ldots, N$. Thus the original basis functions $\varphi(\mathbf{q})$ are augmented by the functions $\left\langle\mathbf{q}|F| \bar{\varphi}_{n}\right\rangle$, which reproduce the $\mathbf{q}$-dependence of the kernel $F\left(\mathbf{q}, \mathbf{q}^{\prime}\right)$. Let us also define the $2 N \times 2 N$ matrix $\tilde{\mathbf{F}}$ via

$$
\tilde{\mathbf{F}}=\left(\begin{array}{cc}
-\mathbf{F} & \mathbf{I} \\
\mathbf{I} & 0
\end{array}\right)
$$

where $\mathbf{F}$ is the $N \times N$ matrix with elements $F_{i j}=\left\langle\varphi_{i}|F| \varphi_{j}\right\rangle$, and $\mathbf{I}$ is the $N \times N$ unit matrix. The Boolean approximation of $F$ can then be written as a rank- $2 N$ separable expansion:

$$
F^{B}=\sum_{n=1}^{2 N} \sum_{m=1}^{2 N}\left|\Phi_{n}>\tilde{\mathbf{F}}_{n m}<\Phi_{m}\right|
$$

In the present paper we use the Boolean approximation of $G_{0}$ to obtain a rank-2N expansion for $G$ :

$$
G^{B}=\sum_{n=1}^{2 N} \sum_{m=1}^{2 N}\left|\Phi_{n}>\left[\left(\tilde{\mathbf{G}}_{0}^{-1}-\tilde{\mathbf{V}}\right)^{-1}\right]_{n m}<\Phi_{m}\right|,
$$

where $(\tilde{\mathbf{V}})_{n m}=\left\langle\Phi_{n}|V| \Phi_{m}\right\rangle$, and

$$
\tilde{\mathbf{G}}_{0}=\left(\begin{array}{cc}
-\mathbf{G}_{\mathbf{0}} & \mathbf{I} \\
\mathbf{I} & 0
\end{array}\right) .
$$

Here $\mathbf{G}_{0}$ is the $N \times N$ matrix consisting of matrix elements $\left\langle\varphi_{n}\left|G_{0}\right| \varphi_{m}\right\rangle$, while the $2 N \times 2 N$ matrix $\tilde{\mathbf{V}}$ is composed of four $N \times N$ blocks whose elements are $\tilde{\mathbf{V}}_{n m}=\left\langle\varphi_{n}|V| \varphi_{m}\right\rangle, \tilde{\mathbf{V}}_{n, N+m}=\left\langle\varphi_{n}\left|V G_{0}\right| \varphi_{m}\right\rangle$, $\tilde{\mathbf{V}}_{N+n, m}=\left\langle\varphi_{n}\left|G_{0} V\right| \varphi_{m}\right\rangle$ and $\tilde{\mathbf{V}}_{N+n, N+m}=\left\langle\varphi_{n}\left|G_{0} V G_{0}\right| \varphi_{m}\right\rangle$, with $n=1, \ldots, N$, and $m=1, \ldots, N$. The T-matrix that results from using $G^{B}$ in the Low equation is denoted as $T^{B}$.

\section{Reduced Two-Variable Equations}

For central potentials, $V\left(\mathbf{q}, \mathbf{q}^{\prime}\right)$ and $T\left(\mathbf{q}, \mathbf{q}^{\prime}\right)$ depend only on $q, q^{\prime}$ and $x_{q q^{\prime}}$. Here, $x_{q q^{\prime}}$ denotes the cosine of the angle between vectors $\mathbf{q}$ and $\mathbf{q}^{\prime}$. If we denote the polar and azimuthal angles of the momentum vectors $\mathbf{q}$ by $\theta$ and $\phi$, respectively, then $x_{q q^{\prime}}=\hat{\mathbf{q}} \cdot \hat{\mathbf{q}}^{\prime}=\cos \theta_{q q^{\prime}}=x x^{\prime}+s s^{\prime} \cos \left(\phi-\phi^{\prime}\right)$, where $x=\cos \theta$ and $s=\sqrt{1-x^{2}}$. To emphasize this functional dependence on $x_{q q^{\prime}}$, we will occasionally use the notation $T\left(q, q^{\prime}, x_{q q^{\prime}}\right)$ to stand for $T\left(\mathbf{q}, \mathbf{q}^{\prime}\right)$.

For central potentials, the azimuthal-angle dependence in $T$-matrix elements can be integrated out so that T-LS and G-LS equations become integral equations in two variables. Towards this end, we introduce the averaged momentum states $\mid q x>$ via

$$
\left|q x>=(2 \pi)^{-1 / 2} \int_{0}^{2 \pi} d \phi\right| \mathbf{q}>=(2 \pi)^{-1 / 2} \int_{0}^{2 \pi} d \phi \mid q \theta \phi>.
$$


We next introduce reduced matrix elements of a two-body operator $F$ via

$$
\hat{F}\left(q, x ; q^{\prime}, x^{\prime}\right)=\left\langle q x|F| q^{\prime} x^{\prime}\right\rangle=(2 \pi)^{-1} \int_{0}^{2 \pi} d \phi \int_{0}^{2 \pi} d \phi^{\prime} F\left(\mathbf{q}, \mathbf{q}^{\prime}\right) .
$$

If the operator $F$ is rotationally invariant, its kernel $F\left(\mathbf{q}, \mathbf{q}^{\prime}\right)$ depend on azimuthal angles only through the difference $\phi-\phi^{\prime}$. Therefore, integration over one of the azimuthal angles can be carried out to obtain

$$
\hat{F}\left(q, x ; q^{\prime}, x^{\prime}\right)=\int_{0}^{2 \pi} d \phi F\left(\mathbf{q}, \mathbf{q}^{\prime}\right)=\int_{0}^{2 \pi} d \phi^{\prime} F\left(\mathbf{q}, \mathbf{q}^{\prime}\right) .
$$

This observation allows us to integrate Eq. (2) over $\phi$ to obtain the reduced two-variable T-LS equation

$$
\begin{aligned}
\hat{T}\left(q, x ; q^{\prime}, x^{\prime}\right)= & \hat{V}\left(q, x ; q^{\prime}, x^{\prime}\right) \\
& +2 \mu \int_{0}^{\infty} q^{\prime 2} d q^{\prime} \int_{-1}^{1} d x^{\prime} \frac{\hat{V}\left(q, x ; q^{\prime \prime}, x^{\prime}\right) \hat{T}\left(q^{\prime \prime}, x^{\prime \prime} ; q^{\prime}, x^{\prime}\right)}{q_{0}^{2}-q^{\prime \prime 2}+i 0} .
\end{aligned}
$$

In operator form, we write

$$
\hat{T}=\hat{V}+\hat{V} \hat{G}_{0} \hat{T}
$$

which is to be understood as an operator equation in the space of two-variable functions (of $q$ and $x$ ). (Operators, matrix elements and other quantities associated with the reduced representation will be distinguished from those of three-dimensional representation by a caret over the symbol). Of course, reduced versions of G-LS and Low equations follow naturally

$$
\begin{aligned}
& \hat{G}=\hat{G}_{0}+\hat{G}_{0} \hat{V} \hat{G}, \\
& \hat{T}=\hat{V}+\hat{V} \hat{G} \hat{V} .
\end{aligned}
$$

The solutions of these equations are sought in a bivariate approximation space $\hat{\mathcal{S}_{\mathcal{A}}}$ spanned by basis functions $\chi_{m}(q, x), i=1,2, \ldots, \hat{N}$. The projector $\hat{\mathcal{P}}$ onto $\hat{\mathcal{S}_{\mathcal{A}}}$ is given as

$$
\hat{\mathcal{P}}=\Sigma_{m=1}^{\hat{N}} \Sigma_{m^{\prime}=1}^{\hat{N}}\left|\chi_{m}>\left(\hat{\boldsymbol{\Delta}}^{-1}\right)_{m, m^{\prime}}<\chi_{m^{\prime}}\right|,
$$

where $\hat{\boldsymbol{\Delta}}$ is the overlap matrix, viz., $\hat{\boldsymbol{\Delta}}_{m, m^{\prime}}=\left\langle\chi_{m} \mid \chi_{m^{\prime}}\right\rangle$. Expressions for reduced T-matrix approximations $\hat{T}^{A}\left(q, x ; q^{\prime}, x^{\prime}\right)$, with $A=O, L, R, I, B$, follow from Eqs. (21)-(24) and (30) with appropriate replacements of three-dimensional states and operators with their reduced two-variable counterparts.

\section{Multivariate Basis Functions}

Most straightforward approach to multivariate approximation is through the tensor-product approach in which a multivariate basis is constructed via tensor products of univariate bases. Refinement of tensor-product approximation is possible via the so-called Boolean combination of projection operators (Blending methods). Of course, the tensor-product methods suffer from the curse of dimensionality. Among various directions taken to overcome the dimensionality problem, the use of radial-functions has become a powerful tool in multivariate approximation theory for approximating scattered data and solving partial differential equations. 


\subsection{Tensor-Product Bases}

The approximation spaces $\hat{\mathcal{S}}_{A}$ and $\mathcal{S}_{A}$ are constructed as tensor products of univariate spaces: $\hat{\mathcal{S}}_{A}=\mathcal{S}_{q} \otimes \mathcal{S}_{x}$ and $\mathcal{S}_{A}=\mathcal{S}_{q} \otimes \mathcal{S}_{x} \otimes \mathcal{S}_{\phi}$. Here the space $\mathcal{S}_{q}$ is $N_{q}$-dimensional and spanned by basis functions $\left\{u_{i}(q), i=\right.$ $\left.1,2, \ldots, N_{q}\right\}$. The space $\mathcal{S}_{x}$ is $N_{x}$-dimensional and spanned by $\left\{v_{j}(x), j=1,2, \ldots, N_{x}\right\}$. Similarly, the space $\mathcal{S}_{\phi}$ is $N_{\phi}$-dimensional and and spanned by $\left\{w_{k}(\phi), k=1,2, \ldots, N_{\phi}\right\}$. Hence, $\hat{\mathcal{S}}_{A}$ is of dimension $\hat{N}=N_{q} N_{x}$, and spanned by the tensor-product basis $\left\{\chi_{i j}(q, x) \equiv u_{i}(q) v_{j}(x)\right\}$. On the other hand, the threevariable space $\mathcal{S}_{A}$ is of dimension $N=N_{q} N_{x} N_{\phi}$, and spanned by the tensor-product basis $\left\{\varphi_{i j k}(q, x, \phi) \equiv\right.$ $\left.u_{i}(q) v_{j}(x) w_{k}(\phi)\right\}$.

The basis sets in the $q, x$ and $\phi$ variables are linearly independent, but not necessarily orthonormal. The overlap matrices $\hat{\boldsymbol{\Delta}}$ and $\boldsymbol{\Delta}$ are a direct-product matrices: $\hat{\boldsymbol{\Delta}}=\boldsymbol{\Delta}_{q} \otimes \boldsymbol{\Delta}_{x}$, and $\boldsymbol{\Delta}=\boldsymbol{\Delta}_{q} \otimes$ $\boldsymbol{\Delta}_{x} \otimes \boldsymbol{\Delta}_{\phi}$, where $\left(\boldsymbol{\Delta}_{q}\right)_{i, i^{\prime}}=\left\langle u_{i} \mid u_{i^{\prime}}\right\rangle,\left(\boldsymbol{\Delta}_{x}\right)_{j, j^{\prime}}=\left\langle v_{j} \mid v_{j^{\prime}}\right\rangle$ and $\left(\boldsymbol{\Delta}_{\phi}\right)_{k, k^{\prime}}=\left\langle w_{k} \mid w_{k^{\prime}}\right\rangle$. The inner products are taken as $\left\langle u_{i} \mid u_{i^{\prime}}\right\rangle=\int_{0}^{\infty} q^{2} d q u_{i}^{*}(q) u_{i^{\prime}}(q), \quad\left\langle v_{j} \mid v_{j^{\prime}}\right\rangle=\int_{-1}^{1} d x v_{j}^{*}(x) v_{j^{\prime}}(x)$ and $\left\langle w_{k} \mid w_{k^{\prime}}\right\rangle=$ $\int_{0}^{2 \pi} d \phi w_{j}^{*}(\phi) w_{j^{\prime}}(\phi)$.

In the tensor product approach we choose the univariate basis functions as local piecewise quadratic polynomials $[27,28]$ defined over a grid, as in the finite element method. The procedure for the construction of the grids for $q, x$ and $\phi$ is the same as decribed earlier in [11]. This procedure generates a computational cutoff $q_{\max }$ for the $q$-variable. These univariate grids are denoted $\left\{q_{i}, i=1,2, \ldots, N_{q}\right\},\left\{x_{j}, j=1,2, \ldots, N_{x}\right\}$, and $\left\{\phi_{k}, k=1,2, \ldots, N_{\phi}\right\}$. Cartesian product $\left\{q_{i}\right\} \times\left\{x_{j}\right\} \times\left\{\phi_{k}\right\}$ generates the interpolation grid for the three-dimensional case, while the cartesian product $\left\{q_{i}\right\} \times\left\{x_{j}\right\}$ gives the interpolation grid for the reduced two-variable case. There is one local quadratic function per grid point for each variable. The local quadratic associated with grid point $q_{i}$ is denoted as $u_{i}(q)$ and it has the cardinal property $u_{i}\left(q_{i^{\prime}}\right)=\delta_{i i^{\prime}}$. These functions are depicted, e.g., in Refs. [27,28,37]. The local basis functions in $x$ and $\phi$ are similarly indexed and have the cardinal property, viz., $v_{j}\left(x_{j^{\prime}}\right)=\delta_{j j^{\prime}}$ and $w_{k}\left(\phi_{k^{\prime}}\right)=\delta_{k k^{\prime}}$.

For our purposes, quadratic interpolates are found to provide sufficient flexibility, although higher order interpolates like cubic hermites or cubic splines [27] could also be used. Use of global univariate bases in place of localized bases is a possibility.

One simple device to build up correlation between univariate variables is to introduce potential-weighted bases (VWB): $\left|\varphi_{n}>=V\right| u_{i} v_{j} w_{k}>$ in the 3-dimensional case, and $\chi_{m}=\hat{V} \mid u_{i} v_{j}>$ for the 2-dimensional case. As will be demonstrated in Sect. 6, the incorporation of the potential into the basis turns out to be very effective in reducing the rank of the separable expansions needed for converged results.

\subsection{Radial Basis Functions}

Radial basis functions(rbf) have recently emerged as popular tools in multivariate interpolation and approximation [17,29-33,38-40]. Scattered-data interpolation and meshless (or mesh-free) methods for the solution of partial differential equations are two main areas where rbf's have drawn considerable attention. In the present work, we demonstrate that rbf's provide a convenient choice for the multivariate bases needed to construct the various projection approximations to the resolvent operators.

The salient features of the rbf approach is as follows: To construct a $D$-variate radial basis, a set of nodes (called rbf centers) are chosen over the computational domain of interest in the $D$-dimensional space $\mathrm{R}^{D}$. This set of nodes do not have to be regularly spaced or have a structured pattern. The distribution and density of nodes can vary over the computational domain as needed. As opposed to the case of finite element bases where a partitioning of computational domain into conforming finite elements is needed, rbf's depend only on the node positions. Topological connectivity of the chosen set of nodes is not needed in the definition of radial functions. One radial function is associated with each node and the basis function centered at a given node depends only on the distance of the field point from that node. This radial dependence of the rbf's has the same functional form $\psi(r)$ for all nodes, where $\psi$ is a univariate positive-definite function, and $r$ is the (scaled) radial distance from the node. As distances between points are relatively easy to calculate in any number of space dimensions, the effort with which radial functions are evaluated is insensitive to the dimension $D$. Also the number of nodes is not directly tied to the dimension $D$. What makes radial functions most useful in multivariate interpolation and approximation is the fact that interpolation/approximation problem becomes relatively insensitive to the dimension $D$. Instead of having to deal with multivariate functions (whose complexity increases with $D$ ), we 
can work with the same univariate function $\psi$ for all cases of $D$. In what follows, we illustrate the details of the rbf approach for the $D=3$ case.

To construct a radial basis for the 3-dimensional momentum space, a set $\left\{\mathbf{q}_{n}, n=1,2, \ldots, N\right\}$ of $N$ distinct centers (or nodes) in three-dimensional momentum space are chosen. We define one rbf, $\varphi_{n}(\mathbf{q})$, associated with each node $\mathbf{q}_{n}$. The $\operatorname{rbf} \varphi_{n}(\mathbf{q})$ is centered at $\mathbf{q}_{n}$ and depends only on the distance from point $\mathbf{q}$ to center $\mathbf{q}_{n}$. Thus each basis function $\varphi_{n}$ is radially symmetric about its center $\mathbf{q}_{n}$. How rapidly an rbf changes with distance from the center can be adjusted individually by introducing a shape parameter $R_{n}$ for each center.

The radial nature of the rbf's is specified through the choice of a univariate positive-definite function $\psi$ defined over $[0, \infty)$. Different types of rbf's follow from different choices for $\psi$. Given a function $\psi$, a set of centers $\left\{\mathbf{q}_{n}\right\}$, and the associated set of shape parameters $\left\{R_{n}\right\}$, the radial basis functions are given as

$$
\varphi_{n}(\mathbf{q})=\psi\left(r_{n}\right)
$$

where

$$
r_{n}=\frac{\left\|\mathbf{q}-\mathbf{q}_{n}\right\|_{2}}{R_{n}}
$$

with $\left\|\mathbf{q}-\mathbf{q}_{n}\right\|_{2}$ being the Euclidean distance between $\mathbf{q}$ and the center $\mathbf{q}_{\mathbf{n}}$. The function $\psi(r)$ may be locally or globally supported. Many choices of both types have been studied in the literature [17,31-33]. In the present work we have considered the following rbf's:

$$
\psi(r)= \begin{cases}e^{-r^{2}} & \text { Gaussian } \\ \left(1+r^{2}\right)^{1 / 2} & \text { Multiquadric (MQ) } \\ \left(1+r^{2}\right)^{-1 / 2} & \text { Inverse Multiquadric (InvMQ) } \\ \left(1+r^{2}\right)^{-1} & \text { Inverse Quadratic (InvQ) } \\ (1-r)_{+}^{4}(1+4 r) & \text { Wendland } \\ \ln [(2+r) /(1+r)] & \end{cases}
$$

Note that Wendland function vanishes for $r \geq 1$. Hence the Wendland function is a compactly supported rbf, while all others in the above list are globally supported.

For the treatment of the reduced-dimension LS equation in variables $q$ and $x$, we choose a set of $\hat{N}$ rbf centers $\left\{\left(q_{m}, x_{m}\right)\right\}$ in the computational $q-x$ domain. The bivariate radial functions $\chi_{m}(q, x)$ are then defined as

$$
\chi_{m}(q, x)=\psi\left(r_{m}\right)
$$

where $r_{m}$ is the scaled Euclidean distance between points $(q, x)$ and $\left(q_{m}, x_{m}\right)$, viz.,

$$
r_{m}=\frac{\sqrt{q^{2}+q_{m}^{2}-2 q q_{m} \cos \alpha}}{R_{n}}
$$

where $\cos \alpha=x x_{m}+\sqrt{1-x^{2}} \sqrt{1-x_{m}^{2}}$.

The question of how to choose the rbf centers and their shape parameters is non-trivial and there are no generally applicable answers [38-40]. In the present work, the Cartesian-product set $\left\{q_{i}, x_{j}, \phi_{k}\right\}$ of grid points used in the previous section to construct the tensor-product finite-element bases is also used as the set of rbf centers. In other words, our rbf centers lie on concentric spheres of radius $q_{i}, i=1, \ldots, N_{q}$. On the sphere of radius $q_{i}$, we have $N_{x} N_{\phi}$ rbf centers that are equally spaced with respect to the $x$ and $\phi$ variables. Note that the point $q_{1}(=0)$ of the $q$-grid requires special attention because all $\left(q_{1}=0, x_{j}, \phi_{k}\right)$ represent the same point (namely, the origin). Therefore, the first point $q_{1}$ of the $q$-grid is shifted from 0 to a small nonzero value. Typically we set $q_{1}=q_{2} / 10$. Similarly, the grid points $x= \pm 1$ of the $x$-grid are offset slightly. This way we generate $N_{q} N_{x} N_{\phi}$ distinct rbf centers.

For the rbf shape parameters $R_{n}$, we have used two schemes: For compact-support rbf's, we set $R_{n}=d R_{n n}$ where $d$ is a scale factor at our disposal and $R_{n n}$ is the distance of the $n$th center from its nearest neighboring center. For global rbf's, we assign an average radius $R_{\text {avg }}$ for each $\operatorname{rbf}$ center $\mathbf{q}_{n} \equiv\left(q_{i}, x_{j}, \phi_{k}\right)$ via $R_{\text {avg }}=$ $\left[4\left(q_{i+1}^{3}-q_{i}^{3}\right) /\left(3 N_{x} N_{\phi}\right]^{1 / 3}\right.$, and set $R_{n}=d R_{a v g}$. Here, $d$ is a scale parameter as before.

For the two-dimensional case, the Cartesian-product set $\left\{q_{i}, x_{j}\right\}$ of grid points in the $\left[0, q_{\max }\right] \times[-1,+1]$ domain used for the construction of tensor-product basis also serves as the set of rbf centers. That is, rbf 
centers lie on concentric circles of radius $q_{i}, i=1, \ldots, N_{q}$. For each $q_{i}$, there are $N_{x}$ rbf centers equally spaced with respect to $x$. Again, the grid point $q_{1}$ of the $q$-grid is shifted from 0 to a small nonzero value (namely, $q_{1}=q_{2} / 10$ ). To each rbf center $\left(q_{i}, x_{j}\right)$, we associate a shape parameter as follows: For compact rbf's, we take $R_{n}=d R_{n n}$, where $R_{n n}$ is the distance to the nearest neighboring center. For global rbf's, on the other hand, we assign an average radius $R_{\text {avg }}$ for each rbf center $\left(q_{i}, x_{j}\right)$ via $R_{\text {avg }}=\left[4 \pi\left(q_{i+1}^{2}-q_{i}^{2}\right) / N_{x}\right]^{1 / 2}$, and set $R_{n}=d R_{\text {avg }}$ for centers located on the circle of radius $q_{i}$. Here, as in the three-dimensional case, the scale factor $d$ can be used to adjust the shape of the rbf's.

\section{Calculations}

Various approximation schemes and multivariable bases discussed in the previous sections have been tested on the Malfliet-Tjon III ( MT-III) model for the two-nucleon potential:

$$
V(r)=V_{R} e^{-\mu_{R} r}-V_{A} e^{-\mu_{A} r}
$$

whose momentum-space representation is given as

$$
V\left(\mathbf{q}, \mathbf{q}^{\prime}\right)=\frac{1}{2 \pi^{2}}\left(\frac{V_{R}}{\left(\mathbf{q}-\mathbf{q}^{\prime}\right)^{2}+\mu_{R}^{2}}-\frac{V_{A}}{\left(\mathbf{q}-\mathbf{q}^{\prime}\right)^{2}+\mu_{A}^{2}} .\right)
$$

For this potential the azimuthal integration in Eq. (4) can be carried out analytically to give

$$
\begin{aligned}
V\left(q, x ; q^{\prime}, x^{\prime}\right)= & \frac{V_{R} / \pi}{\sqrt{\left(q^{2}+q^{\prime 2}-2 q q^{\prime} x x^{\prime}+\mu_{R}^{2}\right)^{2}-4 q^{2} q^{\prime 2}\left(1-x^{2}\right)\left(1-x^{\prime 2}\right)}} \\
& -\frac{V_{A} / \pi}{\sqrt{\left(q^{2}+q^{\prime 2}-2 q q^{\prime} x x^{\prime}+\mu_{A}^{2}\right)^{2}-4 q^{2} q^{\prime 2}\left(1-x^{2}\right)\left(1-x^{\prime 2}\right)}}
\end{aligned}
$$

The parameters for MT-III potential are taken from Ref. [4]: $V_{A}=626.8932 \mathrm{MeV} \mathrm{fm}, V_{R}=1438.723 \mathrm{MeV} \mathrm{fm}$, $\mu_{A}=1.55 \mathrm{fm}^{-1}$ and $\mu_{R}=3.11 \mathrm{fm}^{-1}$. For the two-nucleon calculations, we set nucleon mass and $\hbar$ to unity and take $\mathrm{fm}$ as the unit of length. The nucleon mass adopted yields the conversion factor $1 \mathrm{fm}^{-2}=41.47 \mathrm{MeV}$.

\subsection{Results of Two-Dimensional Calculations}

For general potentials, $V\left(q, x ; q^{\prime}, x^{\prime}\right)$ may not be available analytically. Its numerical generation by applying a suitable quadrature to the integral over the azimuthal angle $\phi$ is quite feasible. For the present potential, the use of a composite 64-point Gauss-Legendre rule for the $\phi$-integral in Eq. (33) produced results that were indistinguishable within 7-8 digits from those of the analytical reduced potential.

Proper treatment of the singular integrals $\left\langle\varphi_{n}\left|G_{0}\right| \varphi_{n^{\prime}}\right\rangle,\left\langle\varphi_{n}\left|V G_{0}\right| \varphi_{n^{\prime}}\right\rangle,\left\langle\varphi_{n}\left|G_{0} V\right| \varphi_{n^{\prime}}\right\rangle$, and $\left\langle\varphi_{n}\left|V G_{0} V\right| \varphi_{n^{\prime}}\right\rangle$ is crucial for the computational implementation of these resolvent approximations. A subtraction procedure and a multi-variable tensor-product quadrature scheme described in detail in Ref. [11] has been used for the numerical treatment of such integrals. Reference solutions against which resolvent approximations are tested have been obtained by either direct quadrature discretization (Nystrom method) of the two-dimensional TLS equation or by Pade resummation of the Born series generated from its iteration. The set of quadrature points typically involved $160 \times 80$ points over the computational $q-x$ domain $\left[0, q_{\max }\right] \times[-1,+1]$. These two-dimensional reference results are stable within at least 6 digits with respect to further refinements of the computational parameters (like $q_{\max }$, number of quadrature points and their distribution).

Tables 1 and 2 show the convergence pattern of the IP-approximation with a tensor-product basis of piecewise quadratic polynomials. Results accurate to 3 digits can be obtained with rather coarse discretizations (with, e.g., $N_{q}=9$ and $N_{x}=11$ ). For convergence within 6 digits after the decimal point, the size of the tensor-product basis must be in the order of $N_{q} \approx 20-30$ and $N_{x} \approx 20-30$. However, if the original tensor product basis $\left\{u_{i}(q) v_{j}(x)\right\}$ is replaced by the potential-weighted basis $\left\{\left\langle q x|V| u_{i} v_{j}\right\rangle\right\}$, convergence pattern is considerably improved.

Table 3 compares the results of OP, LP, Boolean and IP approximations with the largest tensor-product basis $\left(N_{q}=41, N_{x}=41\right)$ employed in this study. Table 4 probes the convergence of various methods with 
Table 1 Convergence of the IP approximation of $\hat{G}_{0}$ using the tensor-product basis of piecewise-quadratic polynomials

\begin{tabular}{|c|c|c|c|c|c|c|c|}
\hline \multirow[t]{2}{*}{ Method } & \multirow[t]{2}{*}{$N_{q}$} & \multicolumn{3}{|c|}{$\operatorname{Re}\left\langle q_{0} x|T| q_{0} x_{0}\right\rangle$} & \multicolumn{3}{|c|}{$\operatorname{Im}\left\langle q_{0} x|T| q_{0} x_{0}\right\rangle$} \\
\hline & & $x=+1.0$ & $\mathrm{x}=0.0$ & $\mathrm{x}=-1.0$ & $\mathrm{x}=+1.0$ & $\mathrm{x}=0.0$ & $\mathrm{x}=-1.0$ \\
\hline \multicolumn{8}{|c|}{$E=150 \mathrm{MeV}$} \\
\hline \multirow[t]{7}{*}{ IP } & 9 & -6.095328 & 0.490984 & 0.239080 & -1.907536 & 0.287980 & 0.367198 \\
\hline & 13 & -6.090332 & 0.491713 & 0.234337 & -1.933994 & 0.285816 & 0.365106 \\
\hline & 17 & -6.092751 & 0.491766 & 0.233976 & -1.937120 & 0.286124 & 0.365652 \\
\hline & 21 & -6.092729 & 0.491776 & 0.233972 & -1.937179 & 0.286100 & 0.365637 \\
\hline & 27 & -6.092767 & 0.491768 & 0.233959 & -1.937229 & 0.286099 & 0.365648 \\
\hline & 33 & -6.092770 & 0.491768 & 0.233959 & -1.937235 & 0.286097 & 0.365648 \\
\hline & 41 & -6.092772 & 0.491768 & 0.233958 & -1.937239 & 0.286097 & 0.365649 \\
\hline \multirow[t]{6}{*}{ IP-VWB } & 9 & -6.097483 & 0.492231 & 0.235879 & -1.928775 & 0.289381 & 0.367841 \\
\hline & 13 & -6.092786 & 0.491770 & 0.233960 & -1.937216 & 0.286102 & 0.365655 \\
\hline & 17 & -6.092782 & 0.491768 & 0.233958 & -1.937247 & 0.286097 & 0.365649 \\
\hline & 21 & -6.092782 & 0.491768 & 0.233958 & -1.937247 & 0.286097 & 0.365649 \\
\hline & 27 & -6.092786 & 0.491770 & 0.233960 & -1.933722 & 0.286102 & 0.365655 \\
\hline & 33 & -6.092782 & 0.491768 & 0.233958 & -1.937247 & 0.286097 & 0.365649 \\
\hline \multirow{2}{*}{\multicolumn{8}{|c|}{$\begin{array}{c}\text { Nystrom } \\
E=400 \mathrm{MeV}\end{array}$}} \\
\hline & & & & & & & \\
\hline \multirow[t]{7}{*}{ IP } & 9 & -6.136830 & 0.465277 & 0.255380 & -1.175239 & 0.108642 & -0.0794744 \\
\hline & 13 & -6.157386 & 0.454539 & 0.248863 & -1.305827 & 0.110026 & -0.0775728 \\
\hline & 17 & -6.163203 & 0.455086 & 0.249271 & -1.310641 & 0.110849 & -0.0776028 \\
\hline & 21 & -6.163002 & 0.454932 & 0.249126 & -1.310805 & 0.110697 & -0.0776746 \\
\hline & 27 & -6.163434 & 0.454939 & 0.249147 & -1.311044 & 0.110759 & -0.0776380 \\
\hline & 33 & -6.163390 & 0.454933 & 0.249139 & -1.311099 & 0.110746 & -0.0776480 \\
\hline & 41 & -6.163455 & 0.454931 & 0.249139 & -1.311075 & 0.110752 & -0.0776432 \\
\hline \multirow[t]{6}{*}{ IP-VWB } & 9 & -6.183678 & 0.465101 & 0.257227 & -1.262780 & 0.114459 & -0.0795470 \\
\hline & 13 & -6.163603 & 0.455166 & 0.249315 & -1.310091 & 0.110823 & -0.0776345 \\
\hline & 17 & -6.163801 & 0.454936 & 0.249144 & -1.311594 & 0.110756 & -0.0776068 \\
\hline & 21 & -6.163807 & 0.454930 & 0.249139 & -1.311640 & 0.110753 & -0.0776419 \\
\hline & 27 & -6.163808 & 0.454930 & 0.249139 & -1.311641 & 0.110753 & -0.0776421 \\
\hline & 33 & -6.163808 & 0.454930 & 0.249139 & -1.311641 & 0.110753 & -0.0776420 \\
\hline Nystrom & & -6.163808 & 0.454930 & 0.249139 & -1.311641 & 0.110753 & -0.0776420 \\
\hline
\end{tabular}

Listed are the on-shell T-matrix elements $\left\langle q_{0} x|T(E)| q_{0} x_{0}\right\rangle$ with $x_{0}=1.0$ at $E=150$ and $E=450 \mathrm{MeV}$. Parameter $N_{q}$ denotes the number of piecewise quadratic polynomials in the $q$ variable. The number $\left(N_{x}\right)$ of piecewise quadratic polynomials in the $x$-variable is 31 for the calculations reported in this table

Table 2 Convergence of the IP approximation of $\hat{G}_{0}$ with respect to the number $\left(N_{x}\right)$ of piecewise quadratic polynomials in the $x$-basis for a fixed $q$-basis consisting of 33 piecewise quadratic polynomials

\begin{tabular}{|c|c|c|c|c|c|c|c|}
\hline \multirow[t]{2}{*}{ Method } & \multirow[t]{2}{*}{$N_{x}$} & \multicolumn{3}{|c|}{$\underline{\operatorname{Re}\left\langle q_{0} x|T| q_{0} x_{0}\right\rangle}$} & \multicolumn{3}{|c|}{$\operatorname{Im}\left\langle q_{0} x|T| q_{0} x_{0}\right\rangle$} \\
\hline & & $\mathrm{x}=+1.0$ & $\mathrm{x}=0.0$ & $\mathrm{x}=-1.0$ & $\mathrm{x}=+1.0$ & $\mathrm{x}=0.0$ & $\mathrm{x}=-1.0$ \\
\hline \multicolumn{8}{|c|}{$E=150 \mathrm{MeV}$} \\
\hline \multirow[t]{3}{*}{ IP } & 11 & -6.091085 & 0.491789 & 0.233958 & -1.935609 & 0.286136 & 0.365613 \\
\hline & 21 & -6.092703 & 0.491769 & 0.233959 & -1.937179 & 0.286098 & 0.365647 \\
\hline & 31 & -6.092770 & 0.491768 & 0.233959 & -1.937235 & 0.286097 & 0.365648 \\
\hline \multirow[t]{3}{*}{ IP-VWB } & 11 & -6.092767 & 0.491768 & 0.233952 & -1.937239 & 0.286097 & 0.365646 \\
\hline & 21 & -6.092782 & 0.491768 & 0.233958 & -1.937247 & 0.286097 & 0.365649 \\
\hline & 31 & -6.092782 & 0.491768 & 0.233958 & -1.937247 & 0.286097 & 0.365649 \\
\hline \multicolumn{2}{|c|}{$\begin{array}{l}\text { Nystrom } \\
E=400 \mathrm{MeV}\end{array}$} & -6.092782 & 0.491768 & 0.233958 & -1.937247 & 0.286097 & 0.365649 \\
\hline \multirow[t]{3}{*}{ IP } & 11 & -6.149251 & 0.454841 & 0.249151 & -1.278361 & 0.110736 & -0.0776603 \\
\hline & 21 & -6.162056 & 0.454930 & 0.249140 & -1.308555 & 0.110743 & -0.0776474 \\
\hline & 31 & -6.163390 & 0.454933 & 0.249139 & -1.311099 & 0.110746 & -0.0776480 \\
\hline \multirow[t]{3}{*}{ IP-VWB } & 11 & -6.161686 & 0.454934 & 0.248812 & -1.308431 & 0.110759 & -0.0781604 \\
\hline & 21 & -6.163799 & 0.454930 & 0.249138 & -1.311631 & 0.110753 & -0.0776423 \\
\hline & 31 & -6.163808 & 0.454930 & 0.249139 & -1.311641 & 0.110753 & -0.0776420 \\
\hline Nystrom & & -6.163808 & 0.454930 & 0.249139 & -1.311641 & 0.110753 & -0.0776420 \\
\hline
\end{tabular}

Shown are the on-shell T-matrix elements $\left\langle q_{0} x|T(E)| q_{0} x_{0}\right\rangle$ with $x_{0}=1.0$ at $E=150$ and $E=400 \mathrm{MeV}$

the size of $x$-basis for a moderate-size $q$-basis $\left(N_{q}=21\right)$. (Results of RP approximation $T^{R}$ are not shown separately because $T^{L}$ and $T^{R}$ come out identical on-shell). The results of OP and LP approximations are clearly inferior to those of the IP approximation. Even with the largest basis, OP and LP approaches do not 
Finite-Rank Multivariate-Basis Expansions

Table 3 Comparison of various resolvent approximations using the tensor-product basis

\begin{tabular}{|c|c|c|c|c|c|c|}
\hline \multirow[t]{2}{*}{ Method } & \multicolumn{3}{|c|}{$\underline{\operatorname{Re}\left\langle q_{0} x|T| q_{0} x_{0}\right\rangle}$} & \multicolumn{3}{|c|}{$\operatorname{Im}\left\langle q_{0} x|T| q_{0} x_{0}\right\rangle$} \\
\hline & $\mathrm{x}=+1.0$ & $\mathrm{x}=0.0$ & $\mathrm{x}=-1.0$ & $\mathrm{x}=+1.0$ & $\mathrm{x}=0.0$ & $\mathrm{x}=-1.0$ \\
\hline \multicolumn{7}{|l|}{$E=150 \mathrm{MeV}$} \\
\hline $\mathrm{OP}$ & -6.088620 & 0.491327 & 0.233246 & -1.940596 & 0.286578 & 0.364989 \\
\hline $\mathrm{OP}+$ iter. & -6.092003 & 0.491939 & 0.234658 & -1.936514 & 0.285725 & 0.364793 \\
\hline LP & -6.090704 & 0.491548 & 0.233602 & -1.938924 & 0.286338 & 0.365321 \\
\hline LP + iter. & -6.093047 & 0.491574 & 0.234244 & -1.936934 & 0.286063 & 0.365561 \\
\hline Boolean & -6.092782 & 0.491768 & 0.233958 & -1.937242 & 0.286097 & 0.365649 \\
\hline Boolean + iter. & -6.092783 & 0.491768 & 0.233958 & -1.937248 & 0.286097 & 0.365649 \\
\hline IP & -6.092780 & 0.491768 & 0.233958 & -1.937245 & 0.286097 & 0.365649 \\
\hline IP + iter & -6.092783 & 0.491768 & 0.233958 & -1.937247 & 0.286097 & 0.365649 \\
\hline Nystrom & -6.092782 & 0.491768 & 0.233958 & -1.937247 & 0.286097 & 0.365649 \\
\hline \multicolumn{7}{|l|}{$E=400 \mathrm{MeV}$} \\
\hline $\mathrm{OP}$ & -6.153884 & 0.453209 & 0.248960 & -1.326018 & 0.112519 & -0.0762300 \\
\hline $\mathrm{OP}+$ iter. & -6.160412 & 0.455191 & 0.249733 & -1.311109 & 0.109069 & -0.0786572 \\
\hline LP & -6.158874 & 0.454077 & 0.249049 & -1.318762 & 0.111647 & -0.0769387 \\
\hline $\mathrm{LP}+$ iter. & -6.163062 & 0.454873 & 0.249140 & -1.311011 & 0.110593 & -0.0776062 \\
\hline Boolean & -6.163733 & 0.454934 & 0.249141 & -1.311413 & 0.110752 & -0.0776439 \\
\hline Boolean + iter. & -6.163812 & 0.454932 & 0.249140 & -1.311627 & 0.110753 & -0.0776431 \\
\hline IP & -6.163698 & 0.454931 & 0.249139 & -1.311477 & 0.110752 & -0.0776433 \\
\hline IP + iter. & -6.163802 & 0.454930 & 0.249139 & -1.311622 & 0.110753 & -0.0776426 \\
\hline Nystrom & -6.163808 & 0.454930 & 0.249139 & -1.311641 & 0.110753 & -0.776420 \\
\hline
\end{tabular}

Shown are the on-shell T-matrix elements $\left\langle q_{0} x|T(E)| q_{0} x_{0}\right\rangle$ with $x_{0}=1.0$ at energies $E=150$ and $E=400 \mathrm{MeV}$. For these calculations, the dimension $\left(N_{q} \times N_{x}\right)$ of the tensor-product approximation space is $41 \times 41=1,681$

go beyond 3-4 digit accuracy. Table 3 also illustrates the commonly known adage that the accuracy of an approximate solution $T^{A}$ can be improved by using it on the righthand side of the LS equation to obtain an iterated solution: $T^{i t e r}=V+V G_{0} T^{A}$.

Results of the Boolean approximation are on par with those of the IP approximation. However, it must be recalled that the basis size involved in the Boolean approach is double the size of the IP method. It involves simultaneous use of the tensor-product basis $\left\{u_{i}(q) v_{j}(x)\right\}$ and the G0-weighted basis $\left\{\left\langle q x\left|G_{0}\right| u_{i} v_{j}\right\rangle\right\}$. Evidently, the incorporation of the singular nature of $G_{0}$ in momentum space (or, equivalently, the asymptotic outgoing-wave behaviour in coordinate space) into the multivariate basis improves the capacity of the basis to represent the full resolvent.

In the calculations reported in Tables 5,6 and 7, the tensor-product basis (of piecewise quadratics) have been replaced by bivariate radial functions. Again IP method emerges as the best among the methods considered. Already with $231 \mathrm{rbf}$ centers in the $q-x$ domain (which corresponds to a cartesian product of $21 q$ nodes and $11 x$ nodes), all radial functions produce results on par or better than those of the tensor-product basis of similar size. Table 7 compares the convergence pattern of OP,LP, Boolean and IP methods with the number of centers for the Wendland basis. It is remarkable that OP and LP approaches perform considerably better with radial functions than with the tensor-product bases, but non-monotonic variation of results with the number of rbf centers is noticeable. Boolean combination of OP, LP and RP methods, however, appear to converge monotonically as the number of rbf centers is increased.

\subsection{Results of Three-Dimensional Calculations}

Resolvent approximations have also been tested in the context of the full three-dimensional G-LS equation. However due to the curse of dimensionality in operation, it has not been possible to push these calculations to convergence. Pade scheme have been used to solve the three-dimensional T-LS equation. Although Pade resummation converges rather rapidly, its accuracy is adversely affected by the fact that relatively small set of quadrature points are used to evaluate the multivariable integrals. For instance, the results labeled as Pade-3D in Table 8 have been calculated by evaluating the three-dimensional integrals with a tensor-product quadrature rule involving 40 points in $q$-variable, 30 points in $x$-variable and 30 points in $\phi$-variable. Results labeled as Nystrom-2D, however, are the accurate reference solutions mentioned in Sect. 6.1.

Table 8 also shows the results of OP, LP and IP methods using a tensor-product basis of piecewise quadratic polynomials with $N_{q}=21, N_{x}=11$ and $N_{\phi}=10$. Thus, the dimension of approximation space is 2,310. 
Table 4 Comparison of various resolvent approximations using the tensor-product basis

\begin{tabular}{|c|c|c|c|c|c|c|c|}
\hline \multirow[t]{2}{*}{ Method } & \multirow[t]{2}{*}{$N_{x}$} & \multicolumn{3}{|c|}{$\underline{\operatorname{Re}}\left\langle q_{0} x|T| q_{0} x_{0}\right\rangle$} & \multicolumn{3}{|c|}{$\operatorname{Im}\left\langle q_{0} x|T| q_{0} x_{0}\right\rangle$} \\
\hline & & $\mathrm{x}=+1.0$ & $\mathrm{x}=0.0$ & $\mathrm{x}=-1.0$ & $\mathrm{x}=+1.0$ & $\mathrm{x}=0.0$ & $\mathrm{x}=-1.0$ \\
\hline \multicolumn{8}{|c|}{$E=150 \mathrm{MeV}$} \\
\hline \multirow[t]{4}{*}{ OP } & 11 & -6.050796 & 0.486775 & 0.226491 & -1.964443 & 0.290271 & 0.359933 \\
\hline & 21 & -6.052422 & 0.486756 & 0.226490 & -1.968109 & 0.290232 & 0.359968 \\
\hline & 31 & -6.052488 & 0.486756 & 0.226490 & -1.968168 & 0.290231 & 0.359968 \\
\hline & 41 & -6.052496 & 0.486756 & 0.226490 & -1.968174 & 0.290231 & 0.359969 \\
\hline \multirow[t]{4}{*}{ LP } & 11 & -6.071318 & 0.489333 & 0.230219 & -1.951354 & 0.288262 & 0.362935 \\
\hline & 21 & -6.072940 & 0.489314 & 0.230220 & -1.952972 & 0.288223 & 0.362969 \\
\hline & 31 & -6.073006 & 0.489313 & 0.230220 & -1.953029 & 0.288223 & 0.362970 \\
\hline & 41 & -6.073014 & 0.489313 & 0.230220 & -1.953035 & 0.288223 & 0.362970 \\
\hline \multirow[t]{4}{*}{ Boolean } & 11 & -6.091313 & 0.491815 & 0.233985 & -1.935286 & 0.286124 & 0.365648 \\
\hline & 21 & -6.092931 & 0.491795 & 0.233982 & -1.936855 & 0.286087 & 0.365682 \\
\hline & 31 & -6.092997 & 0.491796 & 0.233982 & -1.936911 & 0.286086 & 0.365683 \\
\hline & 41 & -6.093005 & 0.491795 & 0.233982 & -1.936917 & 0.286086 & 0.365670 \\
\hline \multirow[t]{4}{*}{ IP } & 11 & -6.091044 & 0.491796 & 0.233971 & -1.935554 & 0.286139 & 0.365602 \\
\hline & 21 & -6.092663 & 0.491767 & 0.233977 & -1.937124 & 0.286101 & 0.365636 \\
\hline & 31 & -6.092729 & 0.491776 & 0.233972 & -1.937179 & 0.286100 & 0.365637 \\
\hline \multirow{2}{*}{\multicolumn{8}{|c|}{$E=400 \mathrm{MeV}$}} \\
\hline & & & & & & & \\
\hline \multirow[t]{4}{*}{$\mathrm{OP}$} & 11 & -6.072871 & 0.443803 & 0.250896 & -1.374743 & 0.121643 & -0.0683724 \\
\hline & 21 & -6.084576 & 0.443905 & 0.250884 & -1.409815 & 0.121666 & -0.0683590 \\
\hline & 31 & -6.085840 & 0.443908 & 0.250884 & -1.412804 & 0.121669 & -0.0683597 \\
\hline & 41 & -6.086065 & 0.443908 & 0.250884 & -1.413292 & 0.121670 & -0.0683598 \\
\hline \multirow[t]{4}{*}{ LP } & 11 & -6.113277 & 0.449542 & 0.250101 & -1.327036 & 0.116526 & -0.0731390 \\
\hline & 21 & -6.125568 & 0.449636 & 0.250089 & -1.359599 & 0.116539 & -0.0731259 \\
\hline & 31 & -6.126885 & 0.449640 & 0.250089 & -1.362333 & 0.116542 & -0.0731265 \\
\hline & 41 & -6.127119 & 0.449640 & 0.250089 & -1.362777 & 0.116542 & -0.0731266 \\
\hline \multirow[t]{4}{*}{ Boolean } & 11 & -6.150681 & 0.455095 & 0.249241 & -1.275314 & 0.110668 & -0.0777760 \\
\hline & 21 & -6.163494 & 0.455185 & 0.249230 & -1.305312 & 0.110675 & -0.0777633 \\
\hline & 31 & -6.164859 & 0.455188 & 0.249230 & -1.307789 & 0.110678 & -0.0777639 \\
\hline & 41 & -6.165102 & 0.455088 & 0.249229 & -1.308187 & 0.110678 & -0.0777639 \\
\hline \multirow[t]{4}{*}{ IP } & 11 & -6.148864 & 0.454839 & 0.249138 & -1.278115 & 0.110686 & -0.0776869 \\
\hline & 21 & -6.161642 & 0.454999 & 0.249126 & -1.308306 & 0.110694 & -0.0776740 \\
\hline & 31 & -6.163002 & 0.454932 & 0.249126 & -1.310805 & 0.110697 & -0.0776746 \\
\hline & 41 & -6.163245 & 0.454932 & 0.249126 & -1.311208 & 0.110697 & -0.0776747 \\
\hline
\end{tabular}

Shown are the on-shell T-matrix elements $\left\langle q_{0} x|T(E)| q_{0} x_{0}\right\rangle$ with $x_{0}=1.0$ for $E=150$ and $E=400 \mathrm{MeV}$. For calculations of this table, $N_{q}=21$ in all cases

Table 5 Performance of various (bivariate) radial basis functions when used in the IP approximation of $\hat{G}_{0}$

\begin{tabular}{|c|c|c|c|c|c|c|}
\hline \multirow[t]{2}{*}{ Method } & \multicolumn{3}{|c|}{$\underline{\operatorname{Re}\left\langle q_{0} x|T| q_{0} x_{0}\right\rangle}$} & \multicolumn{3}{|c|}{$\operatorname{Im}\left\langle q_{0} x|T| q_{0} x_{0}\right\rangle$} \\
\hline & $\mathrm{x}=+1.0$ & $\mathrm{x}=0.0$ & $\mathrm{x}=-1.0$ & $\mathrm{x}=+1.0$ & $\mathrm{x}=0.0$ & $\mathrm{x}=-1.0$ \\
\hline \multicolumn{7}{|l|}{$E=150 \mathrm{MeV}$} \\
\hline Gaussian $(d=\sqrt{10})$ & -6.092764 & 0.491768 & 0.233952 & -1.937232 & 0.286097 & 0.365644 \\
\hline $\mathrm{MQ}(d=1.0)$ & -6.092179 & 0.491755 & 0.234282 & -1.936620 & 0.286040 & 0.365979 \\
\hline $\operatorname{InvMQ}(d=5.0)$ & -6.092731 & 0.491769 & 0.233964 & -1.937212 & 0.286096 & 0.365641 \\
\hline $\operatorname{InvQ}(d=5.0)$ & -6.092755 & 0.491768 & 0.233955 & -1.921122 & 0.284281 & 0.365797 \\
\hline Wendland $(\mathrm{d}=10.0)$ & -6.092570 & 0.491782 & 0.233975 & -1.936910 & 0.286095 & 0.365534 \\
\hline $\ln \frac{2+r^{2}}{1+r^{2}} \quad(d=5.0)$ & -6.092776 & 0.491768 & 0.233955 & -1.937238 & 0.286097 & 0.365647 \\
\hline Nystrom & -6.092782 & 0.491768 & 0.233958 & -1.937247 & 0.286097 & 0.365649 \\
\hline \multicolumn{7}{|l|}{$E=400 \mathrm{MeV}$} \\
\hline Gaussian $(d=\sqrt{10})$ & -6.163473 & 0.454965 & 0.249276 & -1.310618 & 0.110735 & -0.0777593 \\
\hline $\mathrm{MQ}(d=1.0)$ & -6.160253 & 0.454589 & 0.250117 & -1.308697 & 0.110671 & -0.0774024 \\
\hline $\operatorname{IMQ}(d=5.0)$ & -6.163428 & 0.454903 & 0.249062 & -1.311004 & 0.110705 & -0.0775127 \\
\hline $\mathrm{IQ}(d=5.0)$ & -6.163989 & 0.454930 & 0.249137 & -1.311194 & 0.110748 & -0.0776247 \\
\hline Wendland $(\mathrm{d}=10.0)$ & -6.163167 & 0.455062 & 0.249350 & -1.309150 & 0.110791 & -0.0776762 \\
\hline $\ln \frac{2+r^{2}}{1+r^{2}} \quad(d=5.0)$ & -6.163477 & 0.454941 & 0.249184 & -1.311214 & 0.110767 & -0.0777083 \\
\hline Nystrom & -6.163808 & 0.454930 & 0.249139 & -1.311641 & 0.110753 & -0.0776420 \\
\hline
\end{tabular}

Shown are the on-shell T-matrix elements $\left\langle q_{0} x|T(E)| q_{0} x_{0}\right\rangle$ with $x_{0}=1.0$ for $E=150$ and $E=400 \mathrm{MeV}$. The radial functions are defined on a set of 231 distinct centers on the $q-x$ domain $\left[0, q_{\max }\right] \times[-1,+1]$ 
Finite-Rank Multivariate-Basis Expansions

Table 6 Performance of various (bivariate) radial basis functions when used in IP and LP approximations of $\hat{G}_{0}$

\begin{tabular}{|c|c|c|c|c|c|c|c|}
\hline \multirow{2}{*}{\multicolumn{2}{|c|}{ Method basis }} & \multicolumn{3}{|c|}{$\underline{\operatorname{Re}\left\langle q_{0} x|T| q_{0} x_{0}\right\rangle}$} & \multicolumn{3}{|c|}{$\operatorname{Im}\left\langle q_{0} x|T| q_{0} x_{0}\right\rangle$} \\
\hline & & $\mathrm{x}=+1.0$ & $\mathrm{x}=0.0$ & $\mathrm{x}=-1.0$ & $\mathrm{x}=+1.0$ & $x=0.0$ & $x=-1.0$ \\
\hline \multicolumn{8}{|c|}{$E=150 \mathrm{MeV}$} \\
\hline \multirow[t]{4}{*}{ IP } & $\operatorname{Gaussian}(d=\sqrt{10})$ & -6.092663 & 0.491777 & 0.233972 & -1.937247 & 0.286097 & 0.365649 \\
\hline & $\operatorname{InvQ}(d=5.0)$ & -6.092781 & 0.491768 & 0.233958 & -1.937247 & 0.286097 & 0.365649 \\
\hline & Wendland $(\mathrm{d}=10.0)$ & -6.092771 & 0.491775 & 0.233958 & -1.937153 & 0.286094 & 0.365623 \\
\hline & $\ln \frac{2+r^{2}}{1+r^{2}} \quad(d=5.0)$ & -6.092782 & 0.491768 & 0.233958 & -1.937247 & 0.286097 & 0.365649 \\
\hline \multirow[t]{4}{*}{ LP } & Gaussian $(d=\sqrt{10})$ & -6.090884 & 0.491915 & 0.230938 & -1.935244 & 0.286603 & 0.365390 \\
\hline & $\operatorname{InvQ}(d=5.0)$ & -6.092561 & 0.491741 & 0.234375 & -1.937056 & 0.286119 & 0.365823 \\
\hline & Wendland $(\mathrm{d}=10.0)$ & -6.090044 & 0.490727 & 0.235869 & -1.934338 & 0.286532 & 0.363096 \\
\hline & $\ln \frac{2+r^{2}}{1+r^{2}} \quad(d=5.0)$ & -6.092439 & 0.491794 & 0.234088 & -1.937329 & 0.286101 & 0.365614 \\
\hline \multicolumn{8}{|c|}{$E=400 \mathrm{MeV}$} \\
\hline \multirow[t]{4}{*}{ IP } & $\operatorname{Gaussian}(d=\sqrt{10})$ & -6.163838 & 0.454937 & 0.249190 & -1.311469 & 0.110755 & -0.0776373 \\
\hline & $\operatorname{InvQ}(d=5.0)$ & -6.163777 & 0.454931 & 0.249146 & -1.311573 & 0.110754 & -0.0776354 \\
\hline & Wendland $(\mathrm{d}=10.0)$ & -6.163738 & 0.454930 & 0.249085 & -1.311366 & 0.110753 & -0.0777676 \\
\hline & $\ln \frac{2+r^{2}}{1+r^{2}} \quad(d=5.0)$ & -6.163826 & 0.454930 & 0.249115 & -1.311557 & 0.110752 & -0.0776139 \\
\hline \multirow[t]{4}{*}{ LP } & Gaussian $(d=\sqrt{10})$ & -6.174845 & 0.454084 & 0.306404 & -1.308773 & 0.108478 & -0.0820547 \\
\hline & $\operatorname{InvQ}(d=5.0)$ & -6.159844 & 0.454167 & 0.244644 & -1.305525 & 0.109061 & -0.0882137 \\
\hline & Wendland $(\mathrm{d}=10.0)$ & -6.162339 & 0.454877 & 0.249744 & -1.299845 & 0.110222 & -0.0846201 \\
\hline & $\ln \frac{2+r^{2}}{1+r^{2}} \quad(d=5.0)$ & -6.161167 & 0.455008 & 0.249363 & -1.307953 & 0.110606 & -0.0766466 \\
\hline
\end{tabular}

Shown are the on-shell T-matrix elements $\left\langle q_{0} x|T(E)| q_{0} x_{0}\right\rangle$ with $x_{0}=1.0$ at $E=150$ and $E=400 \mathrm{MeV}$. The radial functions are centered on a set of 441 distinct points on the $q-x$ domain $\left[0, q_{\max }\right] \times[-1,+1]$

Table 7 Comparison of OP, LP and Boolean approximations of $\hat{G}_{0}$ using the bivariate Wendland basis

\begin{tabular}{|c|c|c|c|c|c|c|c|}
\hline \multirow[t]{2}{*}{ Method } & \multirow[t]{2}{*}{$M$} & \multicolumn{3}{|c|}{$\operatorname{Re}\left\langle q_{0} x|T| q_{0} x_{0}\right\rangle$} & \multicolumn{3}{|c|}{$\operatorname{Im}\left\langle q_{0} x|T| q_{0} x_{0}\right\rangle$} \\
\hline & & $\mathrm{x}=+1.0$ & $\mathrm{x}=0.0$ & $\mathrm{x}=-1.0$ & $\mathrm{x}=+1.0$ & $\mathrm{x}=0.0$ & $\mathrm{x}=1.0$ \\
\hline \multicolumn{8}{|c|}{$E=150 \mathrm{MeV}$} \\
\hline \multirow[t]{4}{*}{$\mathrm{OP}$} & 231 & -6.091106 & 0.490865 & 0.235448 & -1.937147 & 0.285410 & 0.361145 \\
\hline & 441 & -6.056424 & 0.488286 & 0.218072 & -1.934505 & 0.288241 & 0.353813 \\
\hline & 651 & -6.092499 & 0.489720 & 0.243889 & -1.948270 & 0.286235 & 0.374949 \\
\hline & 861 & -6.088869 & 0.489910 & 0.236200 & -1.918956 & 0.286754 & 0.353752 \\
\hline \multirow[t]{4}{*}{ LP } & 231 & -6.093267 & 0.490788 & 0.236521 & -1.939694 & 0.285304 & 0.365142 \\
\hline & 441 & -6.090044 & 0.490727 & 0.235869 & -1.934338 & 0.286532 & 0.363096 \\
\hline & 651 & -6.093157 & 0.491018 & 0.236820 & -1.939919 & 0.286141 & 0.367178 \\
\hline & 861 & -6.095006 & 0.490847 & 0.238181 & -1.932682 & 0.286864 & 0.362796 \\
\hline \multirow{3}{*}{ Boolean } & 231 & -6.093029 & 0.491778 & 0.233985 & -1.936870 & 0.286123 & 0.365680 \\
\hline & 441 & -6.092854 & 0.491782 & 0.233982 & -1.937190 & 0.286099 & 0.365643 \\
\hline & 651 & -6.092800 & 0.491777 & 0.233982 & -1.937206 & 0.286096 & 0.365659 \\
\hline \multirow[t]{4}{*}{ IP } & 231 & -6.092570 & 0.491782 & 0.233975 & -1.936910 & 0.286094 & 0.365534 \\
\hline & 441 & -6.092771 & 0.491775 & 0.233939 & -1.937153 & 0.286094 & 0.365623 \\
\hline & 651 & -6.092774 & 0.491767 & 0.233965 & -1.937230 & 0.286093 & 0.365652 \\
\hline & 861 & -6.092789 & 0.491765 & 0.233971 & -1.937221 & 0.286094 & 0.365637 \\
\hline \multicolumn{8}{|c|}{$E=400 \mathrm{MeV}$} \\
\hline \multirow[t]{3}{*}{$\mathrm{OP}$} & 231 & -6.194285 & 0.452618 & 0.250896 & -1.374743 & 0.107831 & -0.0971512 \\
\hline & 441 & -6.149205 & 0.455888 & 0.250884 & -1.409815 & 0.106721 & -0.0898881 \\
\hline & 651 & -6.166962 & 0.456883 & 0.250884 & -1.412804 & 0.110950 & -0.0756300 \\
\hline \multirow[t]{4}{*}{ LP } & 231 & -6.176016 & 0.451855 & 0.253027 & -1.299107 & 0.108194 & -0.0847729 \\
\hline & 441 & -6.162339 & 0.454877 & 0.249744 & -1.299845 & 0.110222 & -0.0846201 \\
\hline & 651 & -6.167219 & 0.455428 & 0.253542 & -1.314644 & 0.110682 & -0.0773862 \\
\hline & 861 & -6.165342 & 0.454515 & 0.255816 & -1.304422 & 0.111774 & -0.0789717 \\
\hline \multirow[t]{3}{*}{ Boolean } & 231 & -6.164102 & 0.455095 & 0.249241 & -1.310827 & 0.110892 & -0.0777429 \\
\hline & 441 & -6.164020 & 0.455185 & 0.249230 & -1.310975 & 0.110771 & -0.0776589 \\
\hline & 651 & -6.163696 & 0.455188 & 0.249230 & -1.311352 & 0.110747 & -0.0776342 \\
\hline \multirow[t]{4}{*}{ IP } & 231 & -6.163167 & 0.455062 & 0.249350 & -1.309150 & 0.110791 & -0.0776762 \\
\hline & 441 & -6.163738 & 0.454930 & 0.249085 & -1.311366 & 0.110753 & -0.0776676 \\
\hline & 651 & -6.163701 & 0.454931 & 0.249125 & -1.311543 & 0.110753 & -0.0776384 \\
\hline & 861 & -6.163668 & 0.454926 & 0.249121 & -1.311518 & 0.110744 & -0.0776859 \\
\hline
\end{tabular}

Shown are the on-shell T-matrix elements $\left\langle q_{0} x|T(E)| q_{0} x_{0}\right\rangle$ with $x_{0}=1.0$ for $E=150$ and $E=400 \mathrm{MeV}$. $M$ is the number of rbf centers on the $q-x$ domain $\left[0, q_{\max }\right] \times[-1,+1]$ 
Table 8 Comparison of various approximations for $G_{0}$ in three dimensions using the tensor-product basis with $N_{q}=21, N_{x}=11$, $N_{\phi}=10$

\begin{tabular}{|c|c|c|c|c|c|c|}
\hline \multirow[t]{2}{*}{ Method } & \multicolumn{3}{|c|}{$\underline{\operatorname{Re}\left\langle q_{0} x \phi|T(E)| q_{0} x_{0} \phi_{0}\right\rangle}$} & \multicolumn{3}{|c|}{$\underline{\operatorname{Im}\left\langle q_{0} x \phi|T(E)| q_{0} x_{0} \phi_{0}\right\rangle}$} \\
\hline & $\mathrm{x}=+1.0$ & $\mathrm{x}=0.0$ & $\mathrm{x}=-1.0$ & $\mathrm{x}=+1.0$ & $\mathrm{x}=0.0$ & $\mathrm{x}=-1.0$ \\
\hline \multicolumn{7}{|l|}{$E=150 \mathrm{MeV}$} \\
\hline OP & -6.050835 & 0.486736 & 0.226459 & -1.966466 & 0.290246 & 0.359925 \\
\hline LP & -6.071355 & 0.489295 & 0.234407 & -1.951377 & 0.288254 & 0.362928 \\
\hline IP & -6.091083 & 0.491757 & 0.233940 & -1.935576 & 0.286114 & 0.365595 \\
\hline Pade-3D & -6.092824 & 0.491728 & 0.233923 & -1.937272 & 0.286080 & 0.365641 \\
\hline Nystrom-2D & -6.092782 & 0.491768 & 0.233958 & -1.937247 & 0.286097 & 0.365649 \\
\hline \multicolumn{7}{|l|}{$E=400 \mathrm{MeV}$} \\
\hline OP & -6.072866 & 0.443774 & 0.250872 & -1.374531 & 0.121675 & -0.0683482 \\
\hline LP & -6.113269 & 0.449501 & 0.250068 & -1.326851 & 0.116554 & -0.0731135 \\
\hline IP & -6.148847 & 0.454795 & 0.249100 & -1.277946 & 0.110718 & -0.0776597 \\
\hline Pade-3D & -6.163566 & 0.454879 & 0.249100 & -1.311157 & 0.110788 & -0.0776149 \\
\hline Nystrom-2D & -6.163808 & 0.454930 & 0.249139 & -1.311641 & 0.110753 & -0.0776420 \\
\hline
\end{tabular}

Shown are the on-shell T-matrix elements $\left\langle q_{0} x \phi|T(E)| q_{0} x_{0} \phi_{0}\right\rangle$ with $x_{0}=1.0, \phi=\phi_{0}=0$, at $E=150$ and $E=400 \mathrm{MeV}$

Table 9 Performance of various (trivariate) radial basis functions when used in the inner projection approximation of $G_{0}$

\begin{tabular}{|c|c|c|c|c|c|c|}
\hline \multirow[t]{2}{*}{ Radial function } & \multicolumn{3}{|c|}{$\operatorname{Re}\left\langle q_{0} x \phi|T| q_{0} x_{0} \phi_{0}\right\rangle$} & \multicolumn{3}{|c|}{$\operatorname{Im}\left\langle q_{0} x \phi|T| q_{0} x_{0} \phi_{0}\right\rangle$} \\
\hline & $\mathrm{x}=+1.0$ & $\mathrm{x}=0.0$ & $\mathrm{x}=-1.0$ & $\mathrm{x}=+1.0$ & $\mathrm{x}=0.0$ & $\mathrm{x}=-1.0$ \\
\hline \multicolumn{7}{|l|}{$E=150 \mathrm{MeV}$} \\
\hline Gaussian $(d=\sqrt{10})$ & -6.092817 & 0.491732 & 0.233926 & -1.937268 & 0.286080 & 0.365640 \\
\hline $\mathrm{MQ}(d=1.0)$ & -6.092127 & 0.492149 & 0.234387 & -1.937622 & 0.286505 & 0.365693 \\
\hline $\operatorname{InvMQ}(d=5.0)$ & -6.092800 & 0.491767 & 0.233924 & -1.937273 & 0.286091 & 0.365653 \\
\hline $\operatorname{InvQ}(d=5.0)$ & -6.092832 & 0.491868 & 0.233922 & -1.937288 & 0.286079 & 0.365615 \\
\hline Wendland $(\mathrm{d}=10.0)$ & -6.092811 & 0.491731 & 0.233925 & -1.937268 & 0.286081 & 0.365642 \\
\hline $\ln \frac{2+r^{2}}{1+r^{2}} \quad(d=10.0)$ & -6.092816 & 0.491735 & 0.233927 & -1.937266 & 0.286090 & 0.365640 \\
\hline \multicolumn{7}{|l|}{$E=400 \mathrm{MeV}$} \\
\hline Gaussian $(d=\sqrt{10})$ & -6.163206 & 0.454811 & 0.249074 & -1.310680 & 0.110693 & -0.0777749 \\
\hline $\mathrm{MQ}(d=1.0)$ & -6.158134 & 0.458354 & 0.236632 & -1.302579 & 0.095672 & -0.0861740 \\
\hline $\operatorname{InvMQ}(d=5.0)$ & -6.161537 & 0.454433 & 0.248144 & -1.308317 & 0.110914 & -0.0773247 \\
\hline $\operatorname{InvQ}(d=5.0)$ & -6.159566 & 0.455068 & 0.249082 & -1.305325 & 0.108946 & -0.0752253 \\
\hline Wendland $(\mathrm{d}=10.0)$ & -6.161539 & 0.454866 & 0.249062 & -1.308019 & 0.110791 & -0.0778165 \\
\hline $\ln \frac{2+r^{2}}{1+r^{2}} \quad(d=10.0)$ & -6.163229 & 0.455586 & 0.249017 & -1.310083 & 0.110680 & -0.0777075 \\
\hline
\end{tabular}

Shown are the on-shell T-matrix elements $\left\langle q_{0} x \phi|T(E)| q_{0} x_{0} \phi_{0}\right\rangle$ with $x_{0}=1.0, \phi=\phi_{0}=0$ for two energies, $E=150$ and 400 $\mathrm{MeV}$. The radial functions are centered on a set of 2,310 distinct points on the $q-x-\phi$ domain $\left[0, q_{\max }\right] \times[-1,+1] \times[0,2 \pi]$

At this level of approximation, even the IP method is hard pressed to produce more than 2-3 digit accuracy, especially at the higher energy. Note that, for OP, LP and IP calculations of Tables 8 and 9, the integrals over $\mathbf{q}$ have been evaluated using a $60 \times 30 \times 30$ set of quadrature points over the $q-x-\phi$ domain $\left[0, q_{\max }\right] \times[-1,+1] \times[0,2 \pi]$.

In Table 9, a variety of tri-variate radial basis functions have been used in the three-dimensional IP approximation for $G_{0}$. The number of rbf centers is 2,310. With the possible exception of the Multiquadrics (MQ) basis, all radial functions considered appear to perform better than the tensor-product basis of piecewise quadratics. Note that shape parameter $d$ used in these tests have been chosen in a somewhat haphazard manner. Further improvements could in fact follow from optimization of the parameter $d$ for each type of rbf and for each choice of rbf centers.

\section{Conclusion}

Present study amply demonstrate that resolvent-based methods can be used to directly calculate the full twobody T-matrix without partial wave decomposition. In an earlier article [11], we had approached the same problem from the view point of potential approximations. The methods considered in [11] correspond to various ways of generating separable expansions of the interaction potential in finite approximation spaces. The present article utilizes separable expansions of the free-resolvent obtained by means of several types of 
projection approximations. In both studies, methods based on inner-projections stand out as the most efficient scheme. In fact, if the same tensor-product bases of piecewise quadratic polynomials is used to form innerprojection approximations $V^{I}$ and $G_{0}^{I}$, the T-matrix results converge faster for the present resolvent-based scheme than the potential-based scheme of Ref. [11].

Of the 5 types of projections considered, approximations of $G_{0}$ via inner projection and Boolean projection perform more satisfactorily than the others. In connection with IP approximation, this finding in multi-variable context is in line with the similar observations in earlier studies of Schwinger-type variational methods in the context of partial-wave LS integral equations in single variable [14,24]. Some insight into this finding can be gained by looking at the coordinate-space kernels of $G_{0}^{I}$ and $G_{0}^{B}$. Both $\left\langle\mathbf{r}\left|G_{0}^{I}\right| \mathbf{r}^{\prime}\right\rangle$ and $\left\langle\mathbf{r}\left|G_{0}^{B}\right| \mathbf{r}^{\prime}\right\rangle$ have the correct behaviour at asymptotic radial distances $r$ or $r^{\prime}$. In contrast, the outer and one-sided projections are deficient in this regard. The rank of the Boolean projection, however, is twice the rank of inner projection, and the computational implementation of the Boolean approach is inherently more involved.

Of paramount importance in multidimensional calculations is how to construct the approximation space. Tensor-product approach is straightforward, but is ultimately handicapped by the lack of correlation or entanglement between univariate variables used construct the multivariate basis. This in turn gives rise to the infamous "curse of dimensionality". To cope with the dimension problem, we need ways of introducing entanglement of variables into the basis functions. Even the selection of variables may make a difference: We used $(q, \cos \theta, \phi)$, but $(q, \theta, \phi)$ or even cartesian components $(x, y, z)$ could be used to build the tensor-product basis. (In fact the Gaussian rbf can be considered as a product of three univariate Gaussians).

In the present study, the incorporation of the potential into the tensor-product finite-element basis has been found to significantly improve the performance of the IP method. By this simple device, variables $q$ and $x$ are entagled, and basis functions become more appropriate for the context of the LS equation. Incidentally, this idea of $V$-weighted basis (VWB) has its counterpart in Ref. [11] in the form of $G_{0}$-weighted basis. In a way the idea behind the blending-function methods [16-20] in the context of multivariate interpolation is similar: the sections (or cuts) of a multivariate-function are used as basis functions to form a finite-rank expansion of that multivariate function. We note that Pade method of solving T-LS equation also involves a similar idea, namely, a multivariate basis is generated by applying the powers of the kernel $V G_{0}$ on the initial momentum state $\mid \mathbf{q}_{0}>$. Results of the present article, as well as those of [11], show that more effective bases may be generated by transforming a primitive basis under the action of operators like $V, G_{0}, V G_{0}$ or $G_{0} V$, depending on the context.

The use of the radial basis functions has emerged during the last two decades as an alternative to tensorproduct bases. Their popularity seems to have further increased with the advent of local compact-support rbf's like Wendland functions $[31,38,39]$. We have tested several types of rbf's to solve the G-LS equation and the results are very encouraging. We believe that these rbf results can further be improved by optimizing the distribution of the rbf centers and the shape parameter associated with each center. Although the set of rbf centers does not have to be on a regular grid, in our implementation we have used the same cartesian-product grid of the tensor-product approach. Optimal choice of rbf centers is a difficult issue that needs to be studied further. Also the shape parameters have not been optimized thoroughly in our calculations. In principle, the optimal shape parameter would vary with number and distribution of rbf centers. There are also many more types of rbf's than we considered in this study.

The most appealing feature of radial functions is the fact that multivariate interpolation/approximation problem becomes insensitive to the dimension. Node placement does not have to be regular and number of nodes (hence the basis size) does not necessarily grow with the number of variables as fast as it does for tensor-product bases. Therefore, radial basis functions are likely to become practical and powerful tools to solve multivariate integral equations of few-body scattering problems without resorting to angular-momentum decompositions.

For instance, three-particle Faddeev equations as elaborated in Refs. [41-43] without invoking partial wave expansion are integral equations in 5 variables. Nystrom method of quadrature discretization is impractical in this case because number of quadrature points needed over this 5-dimensional computational domain can get prohibitively high. Currently these equations are solved via Pade resummation of the Neumann series [42]. (It is interesting to note that the Pade approach is also a dimension-insensitive method).

As an alternative to the Pade approach, one could expand the 3-body amplitudes of interest in a basis of 5-variate radial functions and then use either collocation or Galerkin approach in the Faddeev equations to obtain a system of algebraic equations for the expansion coefficients. Matrix elements to be calculated would involve multidimensional integrals similar to the ones encountered in the Pade method. If one can come up with efficient ways of choosing a set of rbf centers in the 5-dimensional computational domain, dimension- 
independent aspect of the rbf's could make such approaches competitive. Of course, use of rbf's in such a context would not be limited to collocation and Galerkin approaches. By writing Faddeev equations in the standard (matrix) LS form $\mathcal{T}=\mathcal{V}+\mathcal{V} \mathcal{G}_{0} \mathcal{T}$ and identifying the "potential" $\mathcal{V}$ and the "free resolvent" $\mathcal{G}_{0}$ as appropriate (see, e.g., p. 63-66 in Ref. [44]), one can introduce three-particle analogs of all the two-particle methods studied in this paper and in [11]. In particular, the inner projections of $\mathcal{V}$ and $\mathcal{G}_{0}$ in a rbf basis will lead to Schwinger-type variational approximations for $\mathcal{T}$ and, as such, may be expected to better perform than collocation or Galerkin methods.

Acknowledgments The author gratefully acknowledges the computation time provided by Prof. B. Tanatar on the computing facility of his research group in the Department of Physics at Bilkent University.

\section{References}

1. Elster, Ch., Thomas, J.H., Glöckle, W.: Two-body T-matrices without angular-momentum decomposition: energy and momentum dependences. Few-Body Syst. 24, 55 (1998)

2. Shertzer, J., Temkin, A.: Direct calculation of the scattering amplitude without-partial wave analysis. Phys. Rev. A 63, 062714 (2001)

3. Caia, G.L., Pascalutsa, V., Wright, L.E.: Solving potential scattering equations without partial wave decomposition. Phys. Rev. C 69, 034003 (2004)

4. Kessler, B.M., Payne, G.L., Polyzou, W.N.: Application of wavelets to singular integral scattering equations. Phys. Rev. C 70, 034003 (2004)

5. Kadyrov, A.S., Bray, I., Stelbovics, A.T., Saha, B.: Direct solution of the three-dimensional Lippmann-Schwinger equation. J. Phys. B 38, 509 (2005)

6. Ramalho, G., Arriaga, A., Peña, M.T.: Solution of the spectator equation for relativistic NN scattering without partial wave expansion. Few-Body Syst. 39, 123 (2006)

7. Rodríguez-Gallardo, M., Deltuva, A., Cravo, E., Crespo, R., Fonseca, A.C.: Two-body scattering without angular-momentum decomposition. Phys. Rev. C 78, 034602 (2008)

8. Rodríguez-Gallardo, M., Deltuva, A., Cravo, E., Crespo, R., Fonseca, A.C.: Two-body scattering without angular-momentum decomposition: fully off-shell T-matrices. Eur. Phys. J. A42, 601 (2009)

9. Kadyrov, A.S., Abdurakhmanov, I.B., Bray, I., Stelbovics, A.T.: Three-dimensional integral-equation approach to protonand antiproton-hydrogen collisions. Phys. Rev. A 80, 022704 (2009)

10. Veerasamy, S., Elster, Ch., Polyzou, W.N.: Two-nucleon scattering without partial waves using a momentum space Argonne V18 interaction. Few-Body Syst. 54, 2207 (2012)

11. Kuruoğlu, Z.C.: Weighted-residual methods for the solution of two-particle Lippmann-Schwinger equation without partialwave decomposition. Few-Body Syst. 55, 69 (2014)

12. Kuruoğlu, Z.C.: Multivariate Bateman method for two-body scattering without partial-wave decomposition. J. Math. Chem. (2014). doi:10.1007/s10910-014-0352-y

13. Atkinson, K.E.: A Survey of Numerical Methods for the Solution of Fredholm Integral Equations of the Second Kind. SIAM, Philadelphia (1976)

14. Adhikari, S.K.: Variational Principles and the Numerical Solution of Scattering Problems. Wiley, New York (1998)

15. Löwdin, P.O.: Linear Algebra for Quantum Theory. Wiley, New York (1998)

16. Cheney, E.W.: Multivariate Approximation Theory: Selected Topics. SIAM, Philadelphia (1986)

17. Cheney, W., Light, W.: A Course in Approximation Theory. AMS, Providence (2009)

18. Nurnberger, G.: Approximation by Spline Functions. Springer, Berlin (1989)

19. Gordon, W.J.: Blending-function methods of bivariate and multivariate interpolation and approximation. SIAM J. Numer. Anal. 8, 158 (1971)

20. Eyre, D.: Solving three-body integral equations with blending functions. J. Comput. Phys. 73, 447 (1987)

21. Sloan, I.H., Brady, J.T.: Variational approach to the on- and off-shell T matrix. Phys. Rev. C 6, 701 (1972)

22. Kuruoğlu, Z.C., Micha, D.A.: Diatomic transition operators: results of $L^{2}$ basis expansions. J. Chem. Phys. 72, 3328 (1980)

23. Newton, R.G.: Scattering Theory of Particles and Waves, 2nd edn. Springer, Berlin (1982)

24. Staszewska, G., Truhlar, D.G.: Convergence of $L^{2}$ methods for scattering problems. J. Chem. Phys. 86, 2793 (1987)

25. Miller, W.H., Jansen op de Haar, B.M.D.D.: A new basis set method for quantum scattering calculations. J. Chem. Phys. 86, 6213 (1987)

26. Sun, Y., Kouri, D.J., Truhlar, D.G.: A comparative analysis of variational methods for inelastic and reactive scattering. Nucl. Phys. A 508, 41c (1990)

27. Prenter, P.M.: Splines and Variational Methods. Wiley, New York (1975)

28. Fletcher, C.A.J.: Computational Galerkin Methods. Springer, New York (1984)

29. Franke, R.: Scattered data interpolation: tests of some methods. Math. Comput. 38, 181 (1982)

30. Kansa, E.J.: Multiquadrics- A scattered data approximation scheme with applications to computational fluid dynamics-I. Surface approximations and partial derivative estimates. Computers Math. Applic. 19, 127 (1990)

31. Wendland, H.: Meshless Galerkin methods using radial basis functions. Math. Comput. 68, 1521 (1999)

32. Zhang, Y.: Solving partial differential equations by meshless methods using radial basis functions. Appl. Math. Comput. 185, 614 (2007)

33. Hu, X.G., Ho, T.S., Rabitz, H., Askar, A.: Solution of the quantum fluid dynamical equations with radial basis function interpolation. Phys. Rev. E. 61, 5967 (2000) 
34. Lovelace, C.: Practical theory of three-particle states. I. Nonrelativistic. Phys. Rev. B 135, 1225 (1964)

35. Coester, F.: Systematic approximations for the single-channel scattering amplitude. Phys. Rev. 133, B1516 (1964)

36. Scadron, M., Weinberg, S., Wright, J.: Functional analysis and scattering theory. Phys. Rev. 135, B202 (1964)

37. Kuruoglu, Z.C., Levin, F.S.: Wave-packet propagation in momentum space: calculation of sharp-energy S-Matrix elements. Phys. Rev. A 46, 2304 (1992)

38. Fasshauser, G.E.: Meshfree Approximation Methods with MATLAB. World Scientific, Singapore (2007)

39. Wendland, H.: Scattered Data Approximation, vol. 17 of Cambridge Monographs on Computational Mathematics. Cambridge University Press, Cambridge (2005)

40. Fasshauser, G.E., Zhang, J.G.: On choosing 'optimal' shape parameters for RBF approximation. Numer. Algorithms 45, 345 (2007)

41. Schadow, W., Elster, Ch., Glöckle, W.: Three-body scattering below breakup threshold: an approach without using partial waves. Few-Body Syst. 28, 15 (2000)

42. Liu, H., Elster, Ch., Glöckle, W.: Three-body scattering at intermediate energies. Phys. Rev. C 72, 054003 (2005)

43. Elster, Ch., Glöckle, W., Witała, H.: A new approach to the 3D Faddeev equation for three-body scattering. Few-Body Syst. 45, 1 (2009)

44. Schmid, E.W., Ziegelmann, H.: The Quantum Mechanical Three-Body Problem. Pergamon Press, Oxford (1974) 